\title{
A review of computational methods applied for identification and quantification of atherosclerotic plaques in images
}

\author{
Danilo Samuel Jodas ${ }^{\mathrm{a}, \mathrm{c}}$, Aledir Silveira Pereira ${ }^{\mathrm{b}}$, João Manuel R.S. Tavares ${ }^{\mathrm{c}, *}$ \\ a CAPES Foundation, Ministry of Education of Brazil, Brasília - DF, 70040-020, Brazil

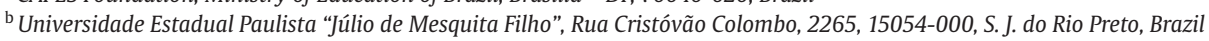 \\ c Instituto de Ciência e Inovação em Engenharia Mecânica e Engenharia Industrial, Faculdade de Engenharia, Universidade do Porto, Rua Dr. Roberto Frias, s/n, \\ 4200-465, Porto, Portugal
}

\section{A R T I C L E I N F O}

\section{Keywords:}

Stroke

Medical imaging

Image analysis

Image segmentation

\begin{abstract}
A B S T R A C T
Evaluation of the composition of atherosclerotic plaques in images is an important task to determine their pathophysiology. Visual analysis is still as the most basic and often approach to determine the morphology of the atherosclerotic plaques. In addition, computer-aided methods have also been developed for identification of features such as echogenicity, texture and surface in such plaques. In this article, a review of the most important methodologies that have been developed to identify the main components of atherosclerotic plaques in images is presented. Hence, computational algorithms that take into consideration the analysis of the plaques echogenicity, image processing techniques, clustering algorithms and supervised classification used for segmentation, i.e. identification, of the atherosclerotic plaque components in ultrasound, computerized tomography and magnetic resonance images are introduced. The main contribution of this paper is to provide a categorization of the most important studies related to the segmentation of atherosclerotic plaques and its components in images acquired by the most used imaging modalities. In addition, the effectiveness and drawbacks of each methodology as well as future researches concerning the segmentation and classification of the atherosclerotic lesions are also discussed.
\end{abstract}

(c) 2015 Elsevier Ltd. All rights reserved.

\section{Introduction}

Cardiovascular diseases represent the main causes of an increasing number of deaths around the world since they impair the heart and vascular system functions. Hence, the early diagnosis of these pathologies is important to minimize clinical cases such as thrombosis, heart attacks, transient ischemic attacks and even the occurrence of strokes. In a broader research study, Mendis, Puska, and Norrving (2011) revealed alarming numbers regarding cardiovascular disease prevention and control: according to the World Health Organization, in 2011 the cardiovascular diseases represented 31\% of the death of people around the world; in 2008, the cardiovascular diseases caused the death of more than 17 millions of people around the world with less than 60-year old. Smoking, lack of physical exercises, inadequate food and excessive consume of alcoholic drinks are the major causes of this disease (Mendis et al., 2011).

\footnotetext{
* Corresponding author. Tel.: +351 225081487; fax: +351 225081445. E-mail addresses: danilojodas@gmail.com (D.S. Jodas), aledir@sjrp.unesp.br (A.S. Pereira), tavares@fe.up.pt (J.M.R.S. Tavares).

URL: http://www.fe.up.pt/ tavares (J.M.R.S. Tavares)
}

One of the main cardiovascular diseases is the atherosclerosis, which occurs as a result of the formation of lipid plaques in the artery wall. The atherosclerosis reduces or occludes the blood flow through the artery, which can cause amaurosis fugax, transient ischemic attack and strokes (Furie, Smimakis, Koroshetz, \& Kistler, 2004; Schadé, 2006; Wiebers, Feigin, \& Brown, 2006).

Technological advances in computerized systems for imaging diagnosis have allowed less invasive ways of analysis and detection of cardiovascular pathologies. Computerized Tomography (CT), Magnetic Resonance Imaging (MRI) and ultrasound are examples of less invasive procedures that have been widely used for evaluating the presence and characteristics of atherosclerotic plaques (Serfaty et al., 2001; de Weert et al., 2006; Widder et al., 1990). Although CT provides better image quality for visualization of the diseases and less invasive procedures compared to the traditional catheter diagnosis, it can be harmful to the health of patients due to the $\mathrm{x}$-rays emission (Brenner \& Hall, 2007; Sodickson et al., 2009). Unlike computerized tomography, ultrasound imaging is a safer procedure since it not exposes the patients to the ionizing radiation. However, the poor image contrast and the speckle noises are the main drawbacks of ultrasound imaging when compared to CT and MRI modalities (Li \& Liu, 2007; Yin \& Liu, 2009; Zhao \& Jolesz, 2014). 
Expedite the carotid endarterectomy is important after onset of symptoms in order to avoid recurrent strokes. In addition, a recurrent stroke can arise within the first two weeks after onset of symptoms and beyond this time a surgical procedure can be inefficient (Salem et al., 2012). Degree of stenosis has been covered as an indicator for evaluating the risks associated with neurological events. In general, patients with degree of stenosis greater or equal than $70 \%$ are selected for carotid endarterectomy in order to prevent the risk of stroke (NASCET, 1991; Warlow, 1991). Although it is a broadly measure related in various studies for selecting patients for carotid endarterectomy, the majority of the patients with significant degree of stenosis remained stroke-free even after years (Lal et al., 2006). In addition, patients with moderate degree of stenosis can also develop symptoms over time (Sztajzel, 2005). Therefore, the analysis of the plaques composition provides the ability of evaluating the progression of atherosclerotic plaques.

Characteristics of echogenicity, texture and surface of atherosclerotic plaques are also addressed in various studies (Geroulakos et al., 1993; Lal et al., 2006; Lovett, Gallagher, Hands, Walton, \& Rothwell, 2004; Steffen, Gray-Weale, Byrne, \& Lusby, 1989; Widder et al., 1990) as indicators of neurological symptoms. Echolucent lesions, heterogenous plaques and ulcerations are described in many studies as the main characteristics associated with high risk for neurological symptoms (Biasi et al., 1998). One of the most used measures to quantitavely evaluate the plaques echogenicity is the Grayscale Median (GSM). In various studies (Biasi et al., 1998; El-Barghouty, Geroulakos, Nicolaides, Androulakis, \& Bahal, 1995; Elatrozy, Nicolaides, Tegos, \& Griffin, 1998; Grogan et al., 2005; Pedro et al., 2000; Salem et al., 2014) the GSM was found to be low in plaques with high risk of neurological symptoms.

Previous studies (Salem et al., 2014; Seeger, Barratt, Lawson, \& Klingman, 1995; Takaya et al., 2006) have addressed the importance of the atherosclerotic plaque burden in evaluating the risks of neurological events. Such components allow the assessment of risks of plaque rupture and embolization, as well as the evaluation of future risks for transient ischemic attacks, amaurosis fugax and strokes. Although visual analysis is a well established method for quantifying the plaque burden, the intra and intervariability between experts may impair the diagnosis. Therefore, development of computational algorithms plays an important role to expedite the assessment of atherosclerotic plaques and avoid the intervariability between experts.

As to expert systems, the identification of atherosclerotic plaques and its main components plays an important role in the evaluation of the disease progression. The classification of such plaques in symptomatic or asymptomatic, for example, is crucial to avoid future cerebral events. In addition, features extracted from the atherosclerotic plaque components allow the development of expert systems to provide medical doctors with an auxiliary tool to automatically classify the occurrence of such events or even the atherosclerotic lesion type. The identification of the lesion type according to the American Heart Association (AHA) classification standard (Herbert, Chandler, \& Dinsmore, 1995) is also a valuable contribution for evaluating the progression of the disease. The composition of the plaque is the basis for classifying the lesion type according to the AHA classification standard. It provides the assessment of the atherosclerotic plaque progression in order to determine the mechanisms that cause its rupture. Hence, the segmentation task represents an essential key in the development of medical decision-making systems that could provide a complementary diagnosis for the atherosclerotic plaques.

A considered number of studies addressing the segmentation of atherosclerotic plaques and its components, as well as the assessment of the occurrence of future cerebral events based on the plaque characteristics, have been proposed. However, the categorization of the main studies is important not only to present an overview of such methodologies, but also to provide the researchers with the employed techniques, the imaging modalities and the effectiveness and drawbacks of each one, as well as future researches to overcome the limitations and improve the accuracy of the current results.

This article presents a review of existing methodologies applied for characterization and quantification of atherosclerotic plaques in ultrasound, CT and MR images. An overview of visual assessment and quantitative analysis applied for characterization of atherosclerotic plaques is presented in Section 2. In addition, a definition about the atherosclerotic plaque components is also presented. Computational algorithms based on image processing techniques, clustering and supervised classification applied for identification and quantification of atherosclerotic plaque components are presented in Section 3. Section 4 is dedicated to discuss advantages and limitations of each methodology. Finally, conclusions and future works are presented in the last section.

\section{Atherosclerotic plaque characterization}

In order to identify the most important characteristics of atherosclerotic plaques associated with neurological events, as well as to quantify the amount of histological components, studies using images acquired from well-known imaging modalities have been presented. Furthermore, the study of the atherosclerotic plaques morphology provides specialists an understanding of its behavior at the moment of treatment and allows to determine whether the plaque will resist the deployment of stents or not (Diethrich, Irshad, \& Reid, 2006). Biasi et al. (1998) reported that dangerous plaques are more predisposed to shed embolic material into the bloodstream when they are manipulated with stent devices. Thus, the identification of safe or dangerous plaques is important to avoid risks prior an angioplasty procedure.

Analysis either using visual classification or computational algorithms have been presented for identification and quantification of atherosclerotic plaques. Computational methods such as image processing techniques and clustering algorithms have been presented in order to automatically outline the atherosclerotic plaque boundaries and classify their main components. In addition, computational algorithms may avoid the intra/inter-variability and the expensive work to manually outline atherosclerotic plaques in images. A review of the most important studies addressing the assessment of the atherosclerotic plaques morphology and histological components identification is presented in this article according to the classification illustrated in Fig. 1.

\subsection{Analysis of atherosclerotic plaques morphology}

Visual analysis of the atherosclerotic plaques echogenicity in ultrasound images has been addressed in several studies for evaluating the presence or absence of neurological symptoms. Echogenicity represents the distribution of the grayscale values within a plaque. Echogenicity is represented by echolucent/anechogenic pattern (dark regions) or echogenic/hyperechogenic pattern (bright regions) (Sztajzel, 2005). In this type of study, an observer performs the visual classification of the plaques based on their echolucent or echogenic pattern. A study performed by Steffen et al. (1989) presented the evaluation of carotid plaques in ultrasound images in order to determine the echogenicity patterns associated with the presence or absence of symptoms. In the study, four types of echogenic and echolucent patterns were used to classify the carotid plaques in symptomatic or asymptomatic groups: type 1 uniformly echolucent; type 2 - predominantly echolucent with small areas of echogenic pattern; type 3 - predominantly echogenic with small echolucent regions; and type 4 - uniformly echogenic. The results shown that types 1 and 2 were more predominant in symptomatic plaques (67\%) and types 3 and 4 were predominant in asymptomatic ones (87\%). Thus, it shows that symptomatic plaques 


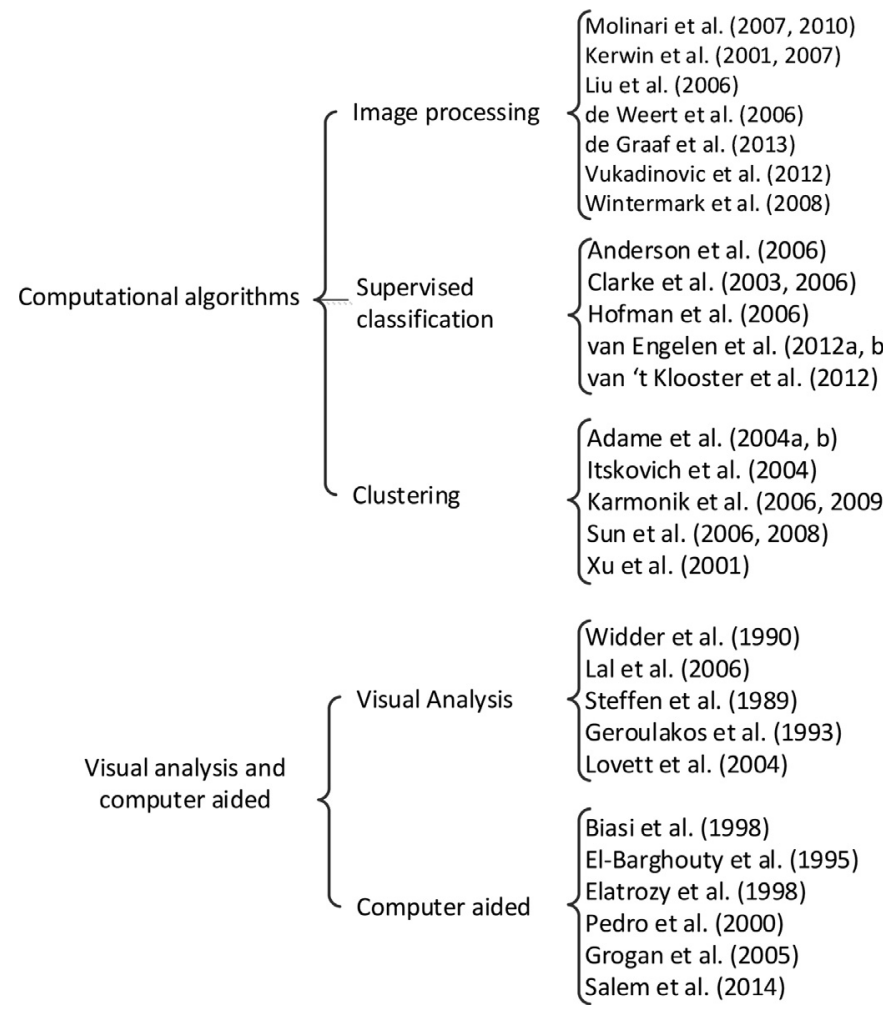

Fig. 1. Classification of the methods reviewed in this article.

are more echolucent than asymptomatic ones. Similar results were achieved by Geroulakos et al. (1993), where the same types were used for classifying the carotid plaques. However, a fifth type was included in order to classify plaques with higher amount of calcification and acoustic shadows. In the study, types 1 and 2 were predominant in symptomatic plaques (81\%), whereas types 3 and 4 were found in asymptomatic ones (59\%). No results concerning the fifth type were presented either for symptomatic or asymptomatic plaques.

Characterization of symptomatic and asymptomatic plaques has been addressed in studies such as the one presented by Lal et al. (2006). Furthermore, morphological characteristics are also evaluated in order to correlate them with the plaque components (Lovett et al., 2004; Widder et al., 1990). In a study proposed by Lal et al. (2006), the identification of intraplaque hemohrrage, large lipid cores and their proximity to the lumen was performed in ultrasound images of carotid plaques in order to correlate them with symptomatic and asymptomatic groups. The authors found that hemorrhage and lipid components were higher in symptomatic plaques, whereas calcium percentage was higher in asymptomatic ones. In addition, lipid core presented higher area and lower distance to the lumen in symptomatic plaques when compared to asymptomatic ones.

Widder et al. (1990) performed an evaluation of the morphological features of carotid plaques in ultrasound images in order to correlate them with the presence of ulcerations and intraplaque hemorrhage. Ultrasound images of the carotid artery have been used to evaluate the border, density and echo structure of plaques. According to the results, regular borders, an echogenic pattern and heterogenous plaques are indicators for discarding the probability of ulcerations and intraplaque hemorrhage.

Lovett et al. (2004) performed a comparison between the surfaces of carotid plaques provided by angiography imaging with histological features. The analysis shown that the increase of the carotid plaque and lipid core components, as well as the decrease of fibrous tissue, are higher in irregular and ulcerated plaques. In addition, irregular and ulcerated plaques were considered $44 \%$ and $50 \%$ as definitely unstable, respectively, whereas smooth plaques was considered 53\% as definitely stable.

\subsection{Grayscale median of atherosclerotic plaques: a quantitative analysis}

Although visual classification showed good results for characterization of atherosclerotic plaques morphology, the subjective analysis among experts may impair the diagnosis. To overcome this problem, computer-aided methods have been proposed for the efficient quantitative analysis of atherosclerotic plaques.

In order to evaluate the relationship between echogenicity pattern and atherosclerotic plaques with evidence of cerebral infarction, a measure called GSM was introduced by El-Barghouty et al. (1995). The GSM value is used to determine the global plaque echogenicity. A plaque with a GSM value below a certain threshold is considered echolucent and of high risk for neurological symptoms.

In a study conducted by El-Barghouty et al. (1995), the GSM values of 184 carotid plaques in duplex ultrasound images were calculated by using Adobe Photoshop ${ }^{\mathrm{TM}}$ software. The results shown that of the 64 plaques with $G S M>32$, only $11 \%$ were associated with brain infarction, whereas of the 84 plaques with $G S M \leq 32,55 \%$ were associated with brain infarction. In addition, of the 53 plaques associated with brain infarction, $13 \%$ has GSM $>32$ and $87 \%$ has $G S M \leq 32$. Of the 95 plaques not associated with brain infarction, $60 \%$ has GSM > 32 and $40 \%$ has $G S M \leq 32$. In short, the study concludes that echolucent pattern could be considered as high-risk for unstable plaques, whereas echogenic characteristic is associated with stable plaques.

Several studies evaluating the plaques echogenicity with the GSM analysis have been conducted using different threshold values. In addition, other characteristics have been included in order to improve the accuracy of the evaluation. Elatrozy et al. (1998) presented the evaluation of carotid plaques in ultrasound images in order to obtain the most important features associated with ipsilateral hemispheric symptoms. The analysis has been performed in order to measure the GSM and the Percent of Echolucent Pixels (PEP) of carotid plaques, as well as to measure the homogeinity, entropy and contrast. To determine how homogenous is a plaque, the homogeinity has been used. Entropy indicates the dissimilarity of the gray level values into the carotid plaques so that heterogenous plaques have higher entropies. Contrast is a measure that determines the variability of grayscale differences so that large values indicate high variation in the grayscale of the pixels. The results shown that the GSM and the PEP were not statiscally significant for asymptomatic plaques, but significant differences were found for symptomatic plaques. According to the results, more symptomatic plaques were found with GSM $<40(84 \%)$ and PEP > $50(80 \%)$ and multiple regression analysis demonstrated that these feature are the most important predictors for the presence or absence of ipsilateral hemispheric symptoms. In addition, the entropy was the measure that presented statistical significance in differentiating symptomatic from asymptomatic plaques. According to this measure, symptomatic plaques tend to be less heterogenous due to the lower entropy (lower than 2.9).

The relationship between echogenicity and atherosclerotic plaques with evidence of cerebral infarction was addressed in the study of Biasi et al. (1998). In this study, the GSM was calculated as the global echogenicity measure of the plaques in order to find an association with symptoms, evidence of cerebral infarction and degree of stenosis. The results shown that symptomatic plaques have more evidence of cerebral infarction (32\%) when compared to asymptomatic ones (16\%). In addition, plaques with more evidence of cerebral infarction are more echolucent than those with no evidence (40\% vs $9 \%$, respectively). Symptomatic plaques have lower GSM values $(38 \pm 13$ ) when compared to asymptomatic ones $(56 \pm 14)$. No statistical significance was found for GSM and degree of stenosis. 
In the study of Pedro et al. (2000) the GSM, percentage of pixels below the value 40 (echolucent pixels), presence of echogenic cap, plaque disruption, echogenic cap thickness, the percentage of echolucent juxtaluminal region and the percentage of echolucent region were considered in the analysis of carotid plaques in ultrasound images. The results shown that symptomatic plaques associated with Brain Infarction (BI) have low GSM and more echolucent pixels when compared to asymptomatic plaques not associated with BI. For the homogenous characteristic, symptomatic plaques associated with BI shown lower GSM, higher percentage of echolucent pixels and higher surface disruption, whereas the presence of echogenic cap was higher in asymptomatic plaques not associated with BI. For the heterogenous group, symptomatic plaques associated with BI shown lower GSM value and higher echolucent juxtaluminal region. It is important to note that the GSM value was lower in all cases.

Grogan et al. (2005) presented a study which aimed to find the most relevant features associated with symptomatic plaques. GSM was obtained from B-mode ultrasound images (ex-vivo carotid plaques) and preoperative color doppler ultrasound. Calcified and necrotic area, as well as the distance of the necrotic core to the lumen, was calculated from histopathological analysis. The authors found more echolucency, less calcified components and lower GSM value in symptomatic plaques. In addition, the percentage of necrotic area was higher in symptomatic plaques and its distance to the lumen is lower compared to asymptomatic ones. The mean GSM calculated from B-mode ultrasound images shown a value of 41 for symptomatic plaques and 60 for asymptomatic ones. These results are statiscally close to those presented by color doppler, in which the mean GSM with 33.8 is echolucent, whereas the mean GSM with 53.6 is echogenic.

Salem et al. (2014) evaluated patients with low risks for unstable plaques by comparing the ultrasound images and histological analysis. In addition to the GSM value, juxtaluminal black area and plaque area were also considered in the study. A correlation between the characteristics acquired from the ultrasound images and the histological classification (stable/unstable) of the plaques was performed in order to select the most significant association. The results shown that a GSM $<25$, a plaque area $>95 \mathrm{~mm}^{2}$ and a juxtaluminal black area $>6 \mathrm{~mm}^{2}$ are the features associated with unstable plaques.

\subsection{Atherosclerotic plaque components}

An atherosclerotic plaque is formed by components such as lipid core, fibrous tissue, smooth muscle cells, intraplaque hemorrhage and calcifications. The American Heart Association (AHA) lesion type classification is a histological examination that divides the atherosclerotic plaques in categories based on the components within the plaques (Herbert et al., 1995). Thus, a matching of imaging features and the histological examinations provided by this standard aid the physicians in accurately determining the atherosclerotic lesion type. The AHA classification of atherosclerotic plaques based on their components is shown in Table 1.

Table 1

AHA lesion type classification (Herbert et al., 1995).

\begin{tabular}{ll}
\hline Type & Description \\
\hline Type I & Initial lesion \\
Type II & Fatty streak with multiple foam cells layers \\
Type III & Intermediate lesion (preatheroma) \\
Type IV & Atheroma \\
Type Va & Fibroatheroma \\
Type Vb & Calcified (lesion type VII) \\
Type Vc & Fibrotic lesion (lesion type VIII) \\
Type VI & Lesion with surface defect, and/or hematoma-hemorrhage, \\
& and/or thrombotic deposit \\
\hline
\end{tabular}

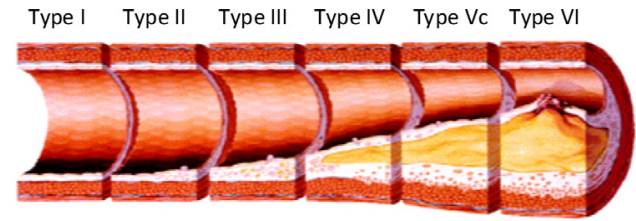

Atherosclerotic plaque timeline

Fig. 2. An example of atherosclerotic plaque progression over time (adapted from Koenig \& Khuseyinova (2007))

A foam cell is a macrophage cell that engulfs fatty components. The accumulation of this type of cells in regions with a large amount of fatty components represents the first stage to the development of atherosclerosis (Herbert et al., 1995). Types I, II and III are considered intermediate lesions, whereas the other types belong to the advanced lesions group. In addition, narrowing of the lumen or obstruction of the blood flow does not occur in these lesion types (Herbert et al., 1995). Lesion types I and II can occur in the childhood, but adults are also likely to these lesions, type III appears after the puberty, whereas type IV appears in the third decade. Subjects after third decade are likely to lesion types V and VI (Herbert et al., 1995). In Fig. 2 is shown an ilustration of the progression of an atherosclerotic disease.

Since the components identified in atherosclerotic plaques are addressed in the AHA lesion type classification, the method can be used in the classification of atherosclerotic lesion types. In fact, several researches addressing the classification of atherosclerotic plaques based on the AHA classification can be found in studies such as those of Cai (2002), Kampschulte et al. (2004) and Saam et al. (2006).

The composition of atherosclerotic plaques has been addressed as an important factor for evaluating the risks of plaque rupture, as well as risks for embolization and neurological events. Histological analysis of the carotid specimens has proved a higher amount of lipid and cholesterol components in symptomatic plaques when compared to asymptomatic ones (Seeger et al., 1995). Unstable plaques associated with majority of strokes contain features such as hemorrhage, large lipid cores, thrombus and plaque inflammation (Salem et al., 2014). Takaya et al. (2006) showed that the presence of thin or ruptured fibrous cap, intraplaque hemorrhage, lipid-rich necrotic core, as well as a larger mean area of intraplaque hemorrhage, larger maximum percentage of lipid-rich necrotic core and maximum wall thickness, were the factors associated with the risk of neurological events.

Identification of atherosclerotic plaque components can be performed in images provided by ultrasound, CT and MRI examinations. The identification of plaque components in ultrasound images is difficult due to their low resolution and artifacts such as noises and acoustic shadows caused by high calcification. Furthermore, the analysis of morphological characteristic of atherosclerotic plaques associated with the presence of components still presents a lack of consensus. As an example, in the study of Bluth et al. (1986) the incidence of intraplaque hemorrhage was higher in heterogeneous plaques (81\%), whereas $96 \%$ of the homogeneous plaques did not present intraplaque hemorrhages. In contrast, the study of SchulteAltedorneburg et al. (2000) showed that hemorrhage was associated with echolucent and homogeneous plaques.

Intravascular Ultrasound (IVUS) is an invasive imaging procedure which allows the evaluation of arterial morphology from within the vessel lumen. Although it is a broadly procedure used for evaluating the arterial diseases, the poor quality of the IVUS images difficult the identification of the plaque components, particularly by automatic algorithms (Diethrich et al., 2006). Intravascular Ultrasound Virtual Histology (IVUS-VH) provides color-mapped images that represent the plaque constitution. The plaque components are identified 
during the IVUS procedure based on the returned frequency of the transducer. That frequency varies depending on the tissue type. These variations allow the real time identification of the components. Several studies (Diethrich et al., 2006; Granada et al., 2007; Nair, 2002) dealing with IVUS-VH examination provide the characterization of the following plaque components: fibrous, fibrofatty, necrotic lipid core and calcifications. Although the IVUS-VH provides an accurate real-time evaluation of the plaques constitution, the procedure is invasive for the patient. Furthermore, IVUS-VH is limited after a stenting procedure because the metal stent is classified as calcification (Diethrich et al., 2006).

Lal et al. (2002) discussed the importance in analyzing the internal structure of carotid plaques in order to characterize their components and select patients at high risk for strokes and atheroembolization. The possibility of identifying carotid plaque components in B-mode ultrasound images is addressed in the study. The methodology called Pixel Distribution Analysis (PDA) was based on the mean grayscale value of subcutaneous fat, muscle, fibrous tissue and calcified structure calculated from control images in order to find these components in carotid plaques. The following mean grayscale values were found for each component: hemorrhage was 2 ( 0 up to 4$)$, lipid was 12 ( 8 up to 26), muscular tissue was 53 (41 up to 76), fibrous tissue was 172 (112 up to 196) and calcium was 221 (211 up to 255). Based on the PDA, the authors found higher levels of blood and lipid components in symptomatic patients $(11.22 \% \pm 3.16$ and $29.38 \% \pm 5.96$, respectively), whereas in asymptomatic patients the calcium and fibromuscular were the components with higher concentration (11.13\% \pm 1.29 and $42.77 \% \pm 5.93$, respectively). The analysis takes into consideration the components with statistical significance. According to the Spearman correlation coefficient, the correlation between PDA and histological analysis for blood, lipid, calcium and fibromuscular components was $0.61,0.77,0.85$ and 0.53 , respectively.

Identification of plaque components on magnetic resonance images has been addressed in some studies. Toussaint, LaMuraglia, Southern, Fuster, and Kantor (1996) presented the ability of T2W images in identifying in vivo and in vitro carotid atherosclerotic plaque components based on the T2 signal. Identification of lipid-rich necrotic cores and intraplaque hemorrhages in MR images performed by Yuan et al. (2001) shown high accuracy when correlated with histological assessment. The study presented by Saam et al. (2005) aimed the evaluation of the ability of MRI exams in quantifying the main components of carotid atherosclerotic plaques. Time-of-Flight (TOF), T1-, T2- and Proton Density Weighted (PDW) images were used to classify the lipid-rich necrotic core, calcification, loose matrix and fibrous tissue in carotid plaques. The components were identified by two radiologists based on the signal intensity (SI) of each MR image. Table 2 indicates the SI values of the plaque components in each MR image.

The results shown a sensitivity of $92 \%, 76 \%, 82 \%$ and $64 \%$ for lipidrich necrotic core, calcification, hemorrhage and loose matrix, respectively. Kappa values were $0.73,0.75,0.71$ and 0.53 , respectively. The correlation was higher for lipid-rich necrotic core and calcification

Table 2

Tissue classification criteria proposed by Saam et al. (2005).

\begin{tabular}{lllll}
\hline & TOF & T1W & PDW & T2W \\
\hline LR/NC with & & & & \\
No or little hemorrhage & 0 & $0 /+$ & $0 /+$ & $-/ 0$ \\
Fresh hemorrhage & + & + & $-/ 0$ & $-/ 0$ \\
Recent hemorrhage & + & + & + & + \\
Calcification & - & - & - & - \\
Loose matrix & 0 & $-/ 0$ & + & + \\
Dense (fibrous) tissue & - & 0 & 0 & 0 \\
\hline
\end{tabular}

The classification into the subgroups is based on the following SIs relative to the adjacent muscle: +, hyperintense; o, isointense; -, hypointense.
(0.75 and 0.74 , respectively) when compared to hemorrhage, loose matrix and fibrous tissue ( $0.66,0.70$ and 0.55 , respectively). The intra and inter-reader reproducibility were also higher for lipid-rich necrotic core and calcification.

According to Chu et al. (2004) the identification of hemorrhage component stages provides the ability of analyzing the progression of the atherosclerotic plaques. Thus, in their study the ability of T1W, T2W, PDW and TOF images in detecting the hemorrhage components stages in atherosclerotic carotid plaques was addressed. The classification of hemorrhages into fresh, recent and old was based on the signal intensity from each MR weighted image and the results were correlated with histological analysis. The sensitivity and specificity found for the hemorrhage areas were $90 \%$ and $74 \%$, respectively; while the hemorrhage stages classification shown moderate kappa value which did not reached 0.80 . The kappa value for one expert was 0.66 , whereas for the other expert was 0.44 . The worst result presented by the second expert can be explained by the difference in the calcium and old hemorrhage determination. These components presented low signal intensity, although the calcification has welldefined borders.

Because thin fibrous cap are likely the most cause of cerebral ischemic diseases in patients with carotid plaques, its evaluation is important to detect possible embolus and hemorrhages. In the study of Yuan (2002) the identification of fibrous cap tissues and its classification into intact and thick, intact and thin and ruptured was performed by evaluating the signal intensity and lumen surface in TOF, T1W, T2W and PDW images. The author found that intact and thick fibrous cap has continuous dark band adjacent to the lumen on TOF images and smooth lumen surface on PDW, T1W and T2W images; intact and thin fibrous cap not presented visible dark band adjacent to the lumen on TOF images, whereas smooth lumen surface is also presented in this fibrous cap type in all other images; finally, the ruptured fibrous cap not present visible dark band adjacent to the lumen on TOF images. Unlike the intact and thin fibrous cap, it was found that ruptured fibrous cap present irregular boundaries on PDW, T1W and $\mathrm{T} 2 \mathrm{~W}$ images.

In a study presented by Watanabe et al. (2014) the evaluation of the ability of Time-of-Flight Magnetic Resonance Angiography (TOF-MRA) images in detecting fibrous cap rupture of atherosclerotic carotid plaques was performed. Fibrous cap status was determined by using the SI of the TOF-MRA. A hypointense band signal with various thickness indicates the presence of fibrous cap, whereas the absence of this signal represents a ruptured fibrous cap. The TOF-MRA images were examined by two radiologists who evaluate the fibrous cap status and reached a consensus about their opinion. In order to evaluate the results, histological specimens obtained from carotid endarterectomy were evaluated by pathologists who identified the fibrous cap rupture and the major components of the plaques. The analysis of histological and MR images shown high sensitivity and moderate specificity (90\% and 69\%, respectively). In addition, the concordance level provided by k-value was moderate (0.59).

\section{Atherosclerotic plaque characterization with computational algorithms}

The aforementioned studies have been proposed to demonstrate the viability of using imaging diagnosis to identify the atherosclerotic plaque components. However, the manual outline of atherosclerotic plaques is a very time consuming task. The development of computational algorithms have been addressed in various studies in order to automatically detect the plaque components and expedite the diagnosis of possible risks for neurological events. In addition, the inter-variability among diagnosis performed by several experts may be avoid by using computational algorithms.

This section presents a review of computational algorithms used for the identification of atherosclerotic plaque components in images 
acquired from the most common imaging examinations. The computational algorithms reviewed in this section are grouped into image processing techniques, clustering algorithms and supervised classification. A summary of each study containing the author names, the publication year, the used imaging modality and the applied algorithm is presented in the final of each subsection.

\subsection{Image processing}

The segmentation is recognized as one of the major tasks in image processing. Usually, segmentation is an essential process to identify structures within an image that can be made in a manual, semiautomatic or automatic way. It constitutes the first step to solve many complex tasks of image processing and analysis, particularly in medical applications. In addition, features extracted, i.e. segmented, from image processing techniques contain important information for the identification and evaluation of risk of diseases either by statistical analysis or by intelligent algorithms. The segmentation of atherosclerotic plaques has been addressed in several studies such as those presented by Loizou, Petroudi, Pantziaris, Nicolaides, and Pattichis (2014); Loizou et al. (2012).

In two studies presented by Molinari et al. (2010); 2007) a method for extraction and characterization of carotid plaques was presented in order to classify them as stable or unstable. In these works, a contrast agent was used to enhance the tissues of the plaques since each component absorbs the contrast agent differently. In the first study, the intima-media thickness and plaques profiles were extracted by using the Completely User-Independent Extraction (CULEX2) algorithm, which was proposed by the same authors (Delsanto et al., 2007). Afterward, the identification of each component of the plaques was performed based on the PDA values (Lal et al., 2002). The results proved that the injection of a contrast agent can improve the areas with echolucent characteristic so that accurate segmentation can be reached. Before the contrast injection, the authors reached a segmentation error of $5 \%$ for stable plaques and $35 \%$ for unstable plaques. However, after the contrast injection the segmentation error for stable plaques was $2 \%$, whereas for those unstable was $8 \%$. In addition, the carotid plaques segmentation error between the manual and automatic segmentation was $1.2 \%$. Regarding the identification of the carotid plaques, the authors found that the presence of $42 \%$ or more of fibrous components represents a stable plaque, whereas an unstable plaque is composed by $25-30 \%$ of hemorrhage and lipid core components.

The same approach applied to the first study to extract the carotid plaque and its components was used in the second study of Molinari et al. (2010). However, the composition of the soft unstable plaques presented by both histological analysis and automatic methods was:

- Hemorrhage: $12 \%$ for the histological analysis and $8 \%$ for the automatic method;

- Lipids: 35\% for the histological analysis and 36\% for the automatic method;

- Fibrous and muscular: 53\% for the histological analysis and 50\% for the automatic method;

- Calcium: no presence for both strategies.

Kerwin et al. (2001) provided a method for the segmentation of atherosclerotic lesions in MR images using grouping of similar pixels and active contours, as well as the classification of the tissue types. In addition, the 3D reconstruction of the artery is performed in order to provide the visualization of the stenosis and the tissues volume. The algorithms used in the study were compiled in a package called Quantitative Vascular Analysis System (QVAS). The 5 steps performed by the proposed framework are:

- Grouping pixels with similar intensities into clusters;
- Place nodes along the boundaries of the regions and connect them with paths in order to determine the initial boundaries. Manual corrections can be necessary;

- Refine the initial contours by using active contours;

- Tissue classification based on the comparison of the mean brightness values from regions of the images and from a compiled library with standard regional mean values of each component;

- Generation of the 3D model.

The method is feed forward, i.e., the results from the previous image are used in the next image in order to reduce the processing time. In addition, this procedure allows the measurement of the lesions volume. The lumen and wall boundaries are used and refined in the next image. Then, the regions from the previous images are used to seek the regions with similar intensities in the next image. In addition, the classification of the tissues in lipid core, fibrous cap or calcification is performed based on the mean brightness value, which change among patients. Regional brightness values and the associated tissue types were defined in a library. Mean brightness value is calculated from the segmented regions and the labeling is performed based on the mean value defined in the library that is closer to that calculated for the regions. In order to minimize the errors caused by the variation of the mean value between patients, the mean values are adapted according to the current tissue.

In a study of Liu et al. (2006) a method called MorphologyEnhanced Probabilistic Plaque Segmentation (MEPPS) for segmentation of carotid plaques based on probability density functions and active contours method was presented. The carotid plaques acquired from T1W, T2W, PDW, TOF and Contrast-Enhanced (CE) imaging were segmented into four tissues: necrotic core, calcification, loose matrix and fibrous tissue. Correction of intensities, normalization, generation of a probability map of each pixel belonging to each tissue type and the application of an active contour in order to delineate the boundaries based on the probabilities maps were the steps performed by the proposed method. The probability map was generated based on the intensity of each contrast weighted image and on two morphological features: distance of the pixel to lumen and wall thickness. Afterward, the active contour method was applied in order to maximize the probabilities and refine the boundaries, as well as avoid noisy artifacts. The image dataset was divided into training set and validation set, being the first one used to generate probabilities maps that represent the probability of each pixel to belong to a tissue type. The results shown a correlation coefficient of $0.78,0.83,0.41$ and 0.82 for necrotic core, calcification, loose matrix and fibrous tissue, respectively. The sensitivity and specificity were higher for MEPPS when compared to manual segmentation, except for calcification. The sensitivity for necrotic core, calcification, loose matrix and fibrous was $0.75,0.65,0.51$ and 0.88 and the specificity was $0.92,0.98,0.97$ and 0.84 , respectively.

In another study performed by Kerwin et al. (2007) the MEPPS algorithm was used to segment the atherosclerotic plaque components. The method begins with the identification of the lumen and outer wall boundaries by using the B-spline snake algorithm (Brigger, Hoeg, \& Unser, 2000). Then, the identification of plaque components, particularly the necrotic core and calcification components, was performed by the MEPPS framework. The normalized wall thickness (area of the wall divided by the total vessel area) and the maximal wall thickness, as well as the average of the necrotic core and calcification from all slices, were calculated for comparison against the manual segmentation. The results shown a high correlation between the manual and automated methods: Normalized wall thickness: 0.90; Maximum wall thickness: 0.84; Necrotic core: 0.86; Calcification: 0.96 . The intraclass correlation coefficient showed good reproducibility of the automatic and manual measurements: Normalized wall thickness: 0.97 and 0.90; Maximum wall thickness: 0.95 and 0.89; Necrotic core: 0.87 and 0.95; and calcification: 0.94 and 
0.98, respectively. Reproducibility was higher for normalized wall thickness, maximum wall thickness and calcification in automatic method, but lower for necrotic core.

Studies performed on Computerized Tomography Angiography (CTA) images have also been proposed for characterization of atherosclerotic plaques. The identification of plaques components using Hounsfield Unit (HU) Attenuation values was described in various studies (de Graaf et al., 2013; Vukadinovic et al., 2011; 2009; de Weert et al., 2006; Wintermark et al., 2008). HU represents the X-Ray attenuation unit used in CT scan examinations (Goldman, 2007). It characterizes the relative density of a substance, i.e. the amount of X-Ray radiation absorbed by each element in the tissue (Bushberg, 2002). A value between -1000 (air, black) and +3000 (dense bone, white) is assigned to each image element (Bushberg, 2002).

A study conducted by de Weert et al. (2006) presented the Hounsfield Unit ranges of fibrous, calcium and lipid-core components in carotid plaques in order to determine the ability of CTA images in identifying such components. The tracing of two regions of interest (ROI) in Multidetector Computer Tomography (MDCT) images was performed by two operators. First, the vessel wall boundary was outlined and the HU ranges of each component were determined. Second, the lumen boundary was outlined and its HU range was determined, as well as the HU of the fibrous tissue near to the lumen. The results shown larger total plaque and calcified areas in MDCT images, whereas fibrous and lipid core areas were smaller when compared to the histological images. According to the authors, the plaque area was larger in MDCT images due to the shrinkage caused by the preparation step of the histological specimens. The lipid-core areas presented the worst results due to the blooming of calcifications area that overshadows area of soft tissue. Thus, it may affect the accuracy and segmentation of lipid-core areas. However, lipid-cores with mildly calcified area shown better results compared to those obtained with hard calcium. It was due to lower blooming effect of the calcium areas. Linear regression showed the following correlations between the proposed method and the analysis of histological specimens: $0.73,0.74$, 0.76 and 0.24 for total plaque area, calcified area, fibrous area and lipid-core area with hard calcium, respectively. Also, the lipid-core area was measured with different levels of calcification. The best correlation was provided by $0-10 \%$ level of calcification $(R=0.77)$.

An automatic method for quantification of atherosclerotic plaques in CTA images of coronary arteries was proposed in de Graaf et al. (2013). Four components were identified: fibrous, fibro-fatty, necrotic core and dense calcium. The extraction of each component was performed by using two approaches: fixed threshold and dynamic threshold. The first approach used fixed HU ranges for extraction of each component, whereas the second one define the cut-off values based on the luminal intensity. The dynamic threshold is based on the fact that the lower luminal intensity, the lower will be the HU value of the plaque. Thus, the $\mathrm{HU}$ of the tissue are defined based on the luminal attenuation. A study performed by Dalager et al. (2011) confirmed the correlation between the attenuation of plaque HU values and the decreasing of the luminal density. Registration of the CTA images with corresponding IVUS images was also performed in order to correlate the results. The results shown that vessel, lumen and plaque volume calculated from CTA images shown high correlation with IVUS results. Regarding the identification of plaque components, a good correlation between CTA and IVUS images was also found as for fixed threshold as for dynamic threshold. However, the correlation coefficient for necrotic core was lower when compared to the other components.

An automatic method for segmentation of the lumen and artery wall boundaries in CTA images was presented in the study of Vukadinovic et al. (2012). Furthermore, the identification of atherosclerotic plaque components based on $\mathrm{HU}$ values was also performed by the proposed method. A set of 40 dataset was used to perform a correlation between the automatic and manual tracing, as well
Table 3

Proposed methodologies for segmentation of atherosclerotic plaque components based on image processing techniques.

\begin{tabular}{|c|c|c|c|}
\hline Author & Year & $\begin{array}{l}\text { Imaging } \\
\text { modality }\end{array}$ & Segmentation method \\
\hline Molinari et al. (2007) & 2007 & Ultrasound & PDA \\
\hline Molinari et al. (2010) & 2010 & Ultrasound & PDA \\
\hline Kerwin et al. (2001) & 2001 & MRI & Active contour and clustering \\
\hline Liu et al. (2006) & 2006 & MRI & Active contour \\
\hline Kerwin et al. (2007) & 2007 & MRI & Active contour \\
\hline de Weert et al. (2006) & 2006 & CTA & $\begin{array}{l}\text { Ranges of Hounsfield Unit } \\
\text { values }\end{array}$ \\
\hline de Graaf et al. (2013) & 2013 & CTA & $\begin{array}{l}\text { Ranges of Hounsfield Unit } \\
\text { values }\end{array}$ \\
\hline Vukadinovic et al. (2012) & 2012 & CTA & Level set and GentleBoost \\
\hline Wintermark et al. (2008) & 2008 & CTA & $\begin{array}{l}\text { Ranges of Hounsfield Unit } \\
\text { values }\end{array}$ \\
\hline
\end{tabular}

as a interobserver analysis. Another set of 90 dataset was used for evaluating the accuracy of the automatic method. The method starts with the segmentation of the lumen boundary using a level set approach. Afterward, the calcium components and the pixels belonging to the vessel region were detected using a GentleBoost framework. Then, calcium and pixels classified in the vessel wall were used to fit an ellipsoid in the vessel wall. From the 40 dataset, the correlation between the manual tracing performed by two observers and the automatic methods showed the worst results for the lipid component: 0.68 (observer 1 ) and 0.79 (observer 2) in lipid volume; 0.52 (observer 1) and 0.57 (observer 2) in lipid percentage. However, the calcium and fibrous components showed moderate and high correlation: 0.97 for both observer in calcium volume, 0.94 for both observer in calcium percentage, 0.94 (observer 1 ) and 0.87 (observer 2) in fibrous volume, and 0.79 (observer 1 ) and 0.73 (observer 2 ) in fibrous percentage. In addition, the fibrous and lipid percentages obtained from 90 databases also presented the worst correlation coefficients: 0.77 and 0.55 , respectively. This was possibly caused by overlaping between the HU ranges of lipid and fibrous tissues.

Wintermark et al. (2008) proposed the identification of carotid plaque components in CTA images based on HU values. Connective tissue, lipid-rich necrotic core, hemorrhage and calcifications were segmented in each $2 \times 2 \mathrm{~mm}^{2}$ region created from a grid delineation in the histological images in combination with micro CT images. Also, the mean Hounsfield attenuation calculated from each corresponding $2 \times 2 \mathrm{~mm}^{2}$ region in the CTA images were used in a linear mixed model in order to obtain the mean Hounsfield attenuation for each plaque component. The results shown an overall agreement of $72.6 \%$ between CTA and histological analysis. The CTA classification of the calcium component is in perfect concordance with the histological analysis. However, the small lipid-core did not present good concordance and it can be assigned to the overlapping of the Hounsfield threshold of the lipid-core and connective tissues. When the large lipid-core is evaluated (equal to or higher than 5 pixels) the results showed good agreement $(k=0.796)$. Hemorrhage also presented good agreement when large amount (equal to or higher than 5 pixels) were considered $(k=0.712)$. The identification of the ulceration also showed good results $(k=0.855)$. The thickness of the fibrous cap showed good correlation between CTA and histological classification $\left(R^{2}=0.77\right)$.

The proposed studies for identification of atherosclerotic plaque components based on image processing techniques are summarized in Table 3.

\subsection{Clustering algorithms}

Clustering represents the partitioning of a dataset in subsets that have similar characteristics. Hence, the similar pixels of an image are partitioned in regions with similar characteristics by using measures 
such as mean, intensity and standard deviation calculated from the image pixels. Clustering algorithms have also been proposed for segmentation of atherosclerotic plaque components (Adame et al., 2004a; 2004b; Itskovich et al., 2004; Karmonik, Basto, \& Morrisett, 2006; Karmonik et al., 2009; Xu, Hwang, \& Yuan, 2001). Since each component is composed by similar intensities, clustering algorithms can be applied to separate the regions of atherosclerotic plaques according to determined criteria.

Adame et al. (2004b) presented a study which the aim was the automatic detection of the boundaries of the lumen and vessel wall based on ellipse fitting and fuzzy clustering. Ellipse fitting was used to detect the outer vessel wall boundary, while fuzzy clustering was applied to identify the lumen boundary and the carotid plaque boundary. Lumen area, outer wall area and fibrous cap thickness were also measured. The definition of an ellipse centered in the lumen center point defined by the user is made to refine the lumen boundary. In addition, fuzzy clustering was used to first segment the lumen region and then the plaque region (represented by the lipid core). In order to perform the experiments, the authors used 50 images acquired from PDW (23) and T1W (27) examinations. The luminal area $\left(\mathrm{mm}^{2}\right)$, outer wall area $\left(\mathrm{mm}^{2}\right)$ and fibrous cap thickness were taken into account in the manual tracing and in the automatic method. The fibrous cap thickness was determined by measuring the mean minimal distance of the lumen and lipid core points. The thickness of the fibrous cap had an acceptable correlation (0.72), whereas the correlations of the lumen and outer wall boundaries were high (0.92 and 0.91, respectively).

In a similar study, Adame et al. (2004a) performed the segmentation of the outer vessel wall, lumen and lipid component in carotid arteries based on fuzzy clustering and ellipse fitting. The first step consists in detecting the outer wall by fitting an ellipse to its boundaries. The regions inside the outer wall was classified into three classes by the fuzzy C-means algorithm: lumen, plaque and wall tissue. The clustering was based on the intensity of T1W or PDW images or even on the combination of the two types of images. The last step comprises the segmentation of the lipid component by using the same clustering algorithm but, this time, two classes were established: wall tissue and lipid component. The automatic method was compared to manual outlines performed by experts and good correlation was found for the three components: 0.94 for lumen, 0.92 for outer wall and 0.76 for fibrous cap thickness.

Itskovich et al. (2004) proposed an approach to segment the coronary plaque components in ex-vivo MR images based on a clustering algorithm. The Spatially Enhance Cluster Analysis (SECA) was used to identify loose fibrous, fibrocellular, lipid-rich necrotic core, thrombus and dense fibrous components. Combined intensities from the T1W, T2W and PWD images were used to initialize the clusters. In order to refine the clusters, the SECA performed the minimization of the chromatic variance and the discontinuity. The first aspect represents the compactness of the cluster and the second one is the rate of the pixels that are not in the same cluster as their neighboring pixels. After the clustering step, the AHA classification of the plaques was performed by experts and compared with those performed by histopathologists in the histological specimens. Regarding the results, the classification based on cluster-analyzed MR images shown good overall agreement with AHA lesion type classification $(k=0.89)$ and it was better when compared to the color composite $(k=0.78)$, T1W ( $k=0.29)$, T2W $(k=0.42)$ and PDW images $(k=0.31)$. In addition, the quantification of the components was performed by calculating its area as the percentage of the total plaque area. When compared to color-spacing, the clustering-analyzed images shown the best correlation with histological analysis. The Pearson correlation for loose fibrous, fibrocellular, lipid-rich necrotic core, thrombus and dense fibrous components were $0.82,0.89,0.79,0.98$ and 0.83 , respectively.

In the study of Karmonik et al. $(2006,2009)$ the identification and quantification of carotid plaques in MR images was performed by the k-means clustering algorithm. The first study was performed on images of ex-vivo carotid plaques, whereas the second one was performed on in-vivo images. The k-means algorithm considered points composed by the combination of the intensity values of the PDW, T1W and T2W MR images. The k-means algorithm classified each point into six classes: fibrous, calcium, thrombus, lipid, normal and background. Comparison between histological section analysis and the corresponding k-means classification was performed in order to evaluate the accuracy of the proposed method. A convergence threshold was used in order to determine the assignment of the points in each cluster. This assignment continues until the sum of the distances of the cluster centers between two iterations is smaller than the convergence threshold. The second study was similar to the first one, but the different approach is the inclusion of in vivo images. The mean differences between histological analysis and the clustering algorithm were $5.8 \pm 4.1,1.5 \pm 1.4,4.0 \pm 2.8,8.2 \pm 10$ and $2.4 \pm 2.2$ for thrombus, calcification, fibrous, normal and lipid components, respectively.

The identification of coronary plaque components based on a spatial penalized fuzzy C-means algorithm and the signal intensities of the components was proposed in the study of Sun et al. (2006). T1W, T2W, PDW and DW (Diffusion Weighted) images acquired from ex-vivo coronary arteries were used to identify and classify the lipid/necrotic core, fibrocellular/fibrous cap, fibrous tissue, thrombus and calcification components. These components were also identified in histological sections of the coronary arteries in order to provide a ground truth for evaluating the proposed method. A spatial penalized fuzzy C-means algorithm was performed to partition the groups corresponding the plaque components. The pixel intensity of each MR weighted image was used by the algorithm. Then, the components were labeled based on their signal intensity pattern. However, the thrombus component was manually outlined on DW images due to its heterogeneous intensity in T1W, T2W and PDW images. Regarding the results, the Pearson correlation coefficient was 0.98 and 0.97 for histology and fresh condition and histology and preserved condition, respectively.

In a similar study, Sun et al. (2008) proposed a method called Prior Information Enhanced Clustering (PIEC) for classification and labeling of the coronary plaque components based on a spatial fuzzy C-means algorithm and $\mathrm{T} 2$ values of the components. As in the previous study, the same spatial fuzzy C-means algorithm was used to segment the regions based on the pixel intensities of T1W, T2W and PDW images. However, only the $\mathrm{T} 2$ values were used to labeling the clusters as calcification, adipose fat, loose matrix, necrotic tissue or fibrocellular component. According to the authors, $\mathrm{T} 2$ values are not dependent on the imaging parameters, but only on the temperature and magnetic field strength. This justifies the use of these values for labeling the plaque components. Regarding the results, the true positive rate for calcification, adipose fat, loose matrix, necrotic tissue and fibrocellular was $88.9,70.6,69.2,94.7$ and 75.0 , respectively.

$\mathrm{Xu}$ et al. (2001) presented the segmentation of carotid plaque components based on a modified mean-shift algorithm (Fukunaga \& Hostetler, 1975). MRI diagnosis was performed in carotid specimens in order to obtain four ex-vivo MR contrast weighted images: T1W, T2W, PDW and TOF. However, T2W images were removed from the data set due to the similarity with PDW images. A modified meanshift algorithm was proposed in order to correctly estimate the initial center of the clusters. In addition, a sphere with dynamic size was considered to minimize the problems of fixed radius used in the original mean-shift algorithm. Calcium, necrotic core, foam cells and fibrous tissues were the components under consideration and the segmentation results were compared to the histological analysis. The misclassification rate values of the calcium, necrotic core, foam cell and fibrous tissue were $2.8,11.1,10.0$ and 4.6 , respectively. In addition, loose fibrous tissue showed the worst results (misclassification rate of 13.5). 
Table 4

Proposed methodologies for segmentation of atherosclerotic plaque components based on clustering algorithms.

\begin{tabular}{llll}
\hline Author & Year & $\begin{array}{l}\text { Imaging } \\
\text { modality }\end{array}$ & Clustering technique \\
\hline Adame et al. (2004b) & 2004 & MRI & Fuzzy clustering \\
Adame et al. (2004a) & 2004 & MRI & Fuzzy clustering \\
Itskovich et al. (2004) & 2004 & MRI & SECA \\
Karmonik et al. (2006) & 2006 & MRI & K-Means \\
Karmonik et al. (2009) & 2009 & MRI & K-Means \\
Sun et al. (2006) & 2006 & MRI & Spatial fuzzy C-Means \\
Sun et al. (2008) & 2008 & MRI & Spatial fuzzy C-Means \\
Xu et al. (2001) & 2001 & MRI & Modified Mean-Shift \\
\hline
\end{tabular}

The proposed studies for identification of atherosclerotic plaque components based on clustering algorithms are summarized in Table 4.

\subsection{Supervised classification of plaque components}

Supervised classification is an important step for solving many pattern recognition problems. In contrast to unsupervised classification, which the classified samples are unlabeled, the supervised classification allows the building of statistic models based on desired outputs provided by experts for each sample. Thus, the classification model may be combined with experience of experts about a certain pattern recognition problem. Supervised classification has been proposed in order to classify the components of atherosclerotic plaques. In addition, the classification of atherosclerotic plaques in symptomatic or asymptomatic was also addressed in several studies (Acharya et al., 2011; 2012; Christodoulou, Pattichis, Pantziaris, \& Nicolaides, 2003; Kyriacou et al., 2006; 2009).

Anderson et al. (2006) presented the segmentation of atherosclerotic plaque components based on predictive models. T1W, T2W and PDW images were acquired from coronary artery specimens. The kmeans clustering algorithm was applied in order to extract the cluster membership of each pixel. In addition, Discrete Cosine Transform and measures extracted from a neighborhood of pixels were also used as input variables by the predictive models. Three predictive models based on an artificial neural network known as Relevant Input Processor Network (RIPNet) were created for a pixel-by-pixel classification of fibrous tissues and lipid components. Each model receives a set of values calculated from the above-mentioned methods. The results showed that the predictive models presented better results when compared to k-means and logistic regression. The results of the predictive models were between $25 \%$ and $30 \%$ better than k-means algorithm and approximately $8 \%$ better than the logistic regression.

In a study of Clarke, Hammond, Mitchell, and Rutt (2003) the identification of fibrous tissue, loose connective tissue, necrotic core and calcification was performed by a minimum distance classifier in eight MR contrast weighted images. Tracing of fibrous, loose connective tissue and necrotic core on histological images were performed by a pathologist in order to provide a ground truth for validating the classifier results. In addition, calcifications were identified on micro CT images. Four ROIs corresponding to each component were outlined in each MR contrast weighted image. The mean value of each ROI was calculated in each MR image. Then, a pixel-by-pixel classification was performed by calculating the Euclidean distance of the pixel to be classified from each mean value belonging to a tissue type so that it is assigned to the tissue associated with the minimum distance. The training of the classifier was performed by using four percent of the total number of pixels. The results shown an overall accuracy of $73.5 \%$. The sensitivity for necrotic core, fibrous tissue, loose connective tissue and calcification were $83.9 \%, 60.4 \%, 65.2 \%$ and $97.6 \%$, respectively. In addition, the reproducibility of the tracing performed by the pathologist was also higher: $96.9 \%, 85.1 \%, 93.8 \%$ and $100 \%$ for necrotic core, fibrous tissue, loose connective tissue and calcification, respectively.

In another study of Clarke, Beletsky, Hammond, Hegele, and Rutt (2006) the identification of carotid plaque components on MR images was performed by using a maximum likelihood classifier. A pixelbased classification was performed in order to assign each one of them into the following five components: fibrous, loose connective tissue, necrotic core, hemorrhage and calcium. The results were compared to histological analysis and micro CT images which served as a gold standard to validate the ability of the MR images in detecting these components. Only $2.5 \%$ of the total plaque area was selected to train the maximum likelihood classifier and these small areas containing the plaque components were obtained from all images. For every pixel of the image, the assignment of its corresponding class was performed by using the maximum likelihood classifier and the comparison of the results was performed by overlapping the MR and histological images. The best overall accuracy was provided by the PDW, T1W and Diffusion-Weighted (DW) images and by the combination of all 8 contrast images used in the study ( $78 \% \pm 15 \%$ for both set of images).

In a study of Hofman et al. (2006) the segmentation of carotid plaque components was performed by using Bayes classifier, k-NN, neural network and Bayes2 classifier. Images acquired from MRI diagnosis were submitted to preprocessing algorithms in order to extract the Ratio Signal Intensity (rSI) of the carotid artery pixels. Also, the carotid artery boundaries were manually outlined by using an inhouse software developed in Mathematica. ROIs defined in all MR weighted images were used in order to obtain the pixels of the homogeneous regions that represent the tissue to be classified. A total of 1811 pixels were extracted from the ROIs and used to train the classifiers. Bayes classifier, k-NN, neural network and Bayes2 classifier were used to classify the pixels into the following four tissue types: fibrous, calcium, hemorrhage and lipid core. Bayes, k-NN and neural network classification were based on the individual rSI of the pixels, whereas the Bayes2 was based on the spatial context of the neighborhood of the pixels. The classification was performed in each MR image slice. The final area of each component was obtained from the sum of each area from each slice divided by the number of slices. The results provided by Bayes, k-NN and Bayes2 classifiers presented reasonably results. The neural network presented the worst results. Calcium component presented the worst results in all classifiers. Although the results were moderated and reasonably, no classifier has reached a correlation coefficient of 0.80 .

A method for segmentation of atherosclerotic plaques in ex-vivo MR images using a linear discriminant classifier was proposed in van Engelen et al. (2012). Thirty-two features extracted from intensities, gradient, Laplacian and Euclidean distances of the voxels to the lumen and vessel wall were used as input for the classifier. The study describes the use of histological images as a ground truth for fibrous and necrotic core, whereas micro CT images were used as ground truth for calcification. The lumen, outer vessel wall and lipid-rich necrotic core were manually drawn on the digitized histological slices, while the calcification was determined by using a Hounsfield threshold value in the micro CT images. The remain component was classified as fibrous tissue. Registration of the ex-vivo MRI and micro CT with histology images were performed in order to obtain a ground truth image with the three components. The results shown an improvement of the accuracy when all 32 features were included. The sensitivity and specificity were $81 \%$ and $97 \%$ for calcification; $85 \%$ and $60 \%$ for fibrous; and $52 \%$ and $89 \%$ for necrotic core, respectively. Percentage of plaque volume of each component was measured and correlated with the ground truth segmentation. The Spearman coefficient for calcification, fibrous and lipid rich necrotic core was $0.86,0.71$ and 0.72 , respectively. 
In another study, van Engelen et al. (2012) described that histological sections are not well aligned with corresponding images due to the deformation caused by the histology processing. In addition, inter and intra-variability, as well as the overlapping between classes, are the most problems of the use of manual segmentation as ground truth. Based on these problems, the authors provided the classification of plaque components (fibrous tissue, lipid-rich necrotic core and calcium) based on the probability of each voxel belongs to a component. The probability was calculated by using the Gaussian blur and the dice overlaping. The main objective of the study was deal with inaccuracies registrations between in vivo and histological data by measuring the probability and dice overlap of each voxel. Thus, samples near to borders or with low dice overlap have lower contribution in the classifier. The ground-truth segmentation was obtained from the manual outline of the components in histological and micro CT images, being the last one used as ground-truth for calcium. After registration of the in-vivo with histologic and micro CT images, each component of the ground-truth segmentation was binarized and blurred with Gaussian filter in order to create soft labels that indicate the probability of each voxel belonging to a component. The soft labels were multiplied with the Dice overlap coefficient calculated from the overlapping between the segmentation of the vessel wall in histology and MR images. It was performed in order to determine the weight of each voxel so that samples close to the boundaries or with low dice overlap coefficient contribute less with the classifier. Twenty three features were extracted from each voxel and together with the soft labels the training was performed by using a linear discriminant classifier. Regarding the results, the Spearman correlation between soft and hard labels per subject was $0.88,0.71$ and 0.75 for calcification, fibrous and necrotic core, respectively. In addition, the soft labels presented lower volume difference when compared with the hard label (manual segmentation of histology and micro CT images) for fibrous and lipid-rich necrotic core components. Per slice, the difference between the classifier results and ground truth using hard labels were $-0.3 \pm 3.6 \%$ for calcification, $9.5 \pm 19.5 \%$ for fibrous tissue and $-9.2 \pm 19.3 \%$ for necrotic tissue. In contrast, the use of soft labels yielded a difference of $-0.6 \pm 3.7 \%$ for calcification, $6.0 \pm 20.8 \%$ for fibrous tissue and $-5.4 \pm 20.4 \%$ for necrotic tissue.

van 't Klooster et al. (2012) proposed the classification of carotid plaque components using a linear discriminant classifier. A group of 60 patients were selected for diagnosis and 3D TOF, T1W, T2W and PDW images were acquired from MRI examinations. Signal intensities of each MR image were used to manually identify the calcification, hemorrhages, lipid core and fibrous components. The manual segmentation was used as a ground truth to evaluate the performance of the automatic method. The automatic classification was performed by a linear discriminant classifier, which receives features such as normalized signal intensity, zero-, first- and second-order derivates, distance to the inner and outer vessel wall and local vessel wall thickness calculated from each pixel in each MR image. The training of the classifier was performed by using images of 20 patients, whereas the validation was performed in images of 40 patients. Regarding the results, the proposed method showed good agreement for presence or absence of each component when compared to the manual classification. An agreement of $80 \%, 82.5 \%$ and $97.5 \%$ was presented for calcification, hemorrhage and lipid core, respectively. In addition, the Pearson correlation coefficient obtained from the volumes calculated by the manual and automatic classifications was $0.80,0.88,0.80$ and 0.10 for hemorrhage, lipid core, fibrous and calcium, respectively. According to the authors, the small number of calcifications compared to other components have in part contributed for the poor results. Also, similar signal intensities of calcifications close to the lumen and other components may explain the poor results.

The reviewed studies for identification of atherosclerotic plaque components based on supervised classification are indicated in Table 5.
Table 5

Reviewed methodologies for segmentation of atherosclerotic plaque components based on supervised classification.

\begin{tabular}{llll}
\hline Author & Year & $\begin{array}{l}\text { Imaging } \\
\text { modality }\end{array}$ & Classifier \\
\hline Anderson et al. (2006) & 2006 & MRI & RIPNet (based on ANN) \\
Clarke et al. (2003) & 2003 & MRI & Minimum distance \\
Clarke et al. (2006) & 2006 & MRI & Maximum likelihood \\
Hofman et al. (2006) & 2006 & MRI & ANN, Bayes and K-NN \\
van Engelen et al. (2012) & 2012 & MRI & Linear discriminant \\
van Engelen et al. (2012) & 2012 & MRI & Linear discriminant \\
van 't Klooster et al. (2012) & 2012 & MRI & Linear discriminant \\
\hline
\end{tabular}

\section{Discussion}

The correct quantification of atherosclerotic plaques is an important key to perform the assessment of risks of neurological events. Studies addressing specifically the segmentation of atherosclerotic plaque components using image processing, clustering algorithms and supervised classification were presented in this review.

Active contours and level sets algorithms have been used in many problems dealing with correct delineation of structural boundaries. Chan-Vese active contours (Chan \& Vese, 2001) have been covered in many researches dealing with boundaries segmentation ( $\mathrm{Ma}$, Tavares, Jorge, \& Mascarenhas, 2010; Michailovich \& Tannenbaum, 2007; Santos et al., 2013; Tang et al., 2012). An advantage of using the Chan-Vese segmentation method concerns the identification of the boundaries without the need of gradient information. Studies such as those presented by Kerwin et al. (2001) and Liu et al. (2006) used active contours algorithms to improve the boundaries of the plaque components.

Clustering have also been proposed for image segmentation. Based on similar characteristics shared by the pixels of the images, clustering algorithms such as k-means and fuzzy C-means have been used in order to partition the images in different regions of interest (Abdel-Dayem \& El-Sakka, 2007; Hooda, Verma, \& Singhal, 2014; Moftah, Elmasry, El-Bendary, Hassanien, \& Nakamatsu, 2012). Although k-means is a well established clustering algorithm that has been proposed in various studies dealing with unsupervised classification, a certain data may belongs to more than one cluster with a certain membership degree (Bezdek, Ehrlich, \& Full, 1984). In many image segmentation problems one intensity value can belongs to more than one cluster due to the overlapping of the grayscale of different regions. Thus, based on the fuzzy concepts the intensity value could have a membership degree in each cluster. In fuzzy clustering each data element can belongs to more than one cluster with a certain membership level, whereas in hard cluster the data can belongs to one cluster only. Fuzzy C-Means was proposed in the study of Adame et al. (2004a, b) for the segmentation of the lipid core in carotid plaques. In the study of Sun et al. (2006); 2008) spatial fuzzy C-Means was proposed as the method for segmentation of the main components in coronary arteries. In addition, results provided by a clustering algorithm can be used to refine the boundaries of the detected regions as shown in the study of Kerwin et al. (2001).

Artificial neural networks, Bayes classifier, k-nearest neighbor and decision tree are examples of algorithms used in supervised classification. Supervised classification also provides the ability of identifying patterns on images based on features extracted from the pixels. Such features are submitted to a classifier which provides an output that represents the class of the presented pattern. However, the classifier model must be constructed by a training set composed by examples along with the corresponding desired outputs. After training, the built model can be used to classify new patterns not belong to the training set. With respect to identification of atherosclerotic plaque components, supervised classification has been proposed in various 
Table 6

Limitations of computational algorithms applied for identification and quantification of atherosclerotic plaques.

\begin{tabular}{|c|c|c|}
\hline Author(s) (year(s)) & Technique(s) & Limitations \\
\hline \multicolumn{3}{|l|}{ Image processing } \\
\hline $\begin{array}{l}\text { Molinari et al. (2010); } \\
\text { 2007) }\end{array}$ & PDA & Redefinition of the gray levels of the components may be necessary \\
\hline Kerwin et al. (2001) & AC & Manual interaction for correcting the edges; No comparisons with radiologists were presented \\
\hline Liu et al. (2006) & AC & Need for a training step for generating the probability maps \\
\hline Kerwin et al. (2007) & AC & The use of the MEPPS algorithm presented the same limitations of the study of Liu et al. (2006) \\
\hline de Weert et al. (2006) & $\mathrm{HU}$ & Difficulty in detecting thrombus and hemorrhage \\
\hline de Graaf et al. (2013) & $\mathrm{HU}$ & It depends on the correct segmentation of the lumen region for calculating the threshold values \\
\hline Vukadinovic et al. (2012) & LS and GB & Overlaping of the HU values difficults the identification of lipids and fibrous tissues \\
\hline Wintermark et al. (2008) & $\mathrm{HU}$ & Variations on the HU values within the $2 \times 2$ grid may impair the correct determination of the mean value \\
\hline \multicolumn{3}{|l|}{ Clustering } \\
\hline Adame et al. (2004a) & FC & Difficults in detecting lipid core close to lumen due to its similarity \\
\hline Adame et al. (2004b) & FC & Difficult in detecting the lipid and fibrous cap due to the similarity of these components in PDW and T1W images \\
\hline Itskovich et al. (2004) & SECA & Calcification was not addressed in the study \\
\hline $\begin{array}{l}\text { Karmonik et al. (2006, } \\
\text { 2009) }\end{array}$ & K-Means & $\begin{array}{l}\text { Choose a converge threshold is computational expensive; K-means algorithm is sensitive to noisy data; Furthermore, } \\
\text { difficults in treating similar data belonging to different clusters are also a problem for k-means algorithm }\end{array}$ \\
\hline Sun et al. (2006) & SFC & The use of the signal intensity is not adequate since it changes with the acquisition parameters \\
\hline Sun et al. (2008) & SFC & Difficults in identifying the loose matrix, fibrocellular and adipose fat due to the similar T2 values of these components \\
\hline Xu et al. (2001) & MMS & The complexity of the Mean Shift algorithm increases when the feature space dimensionality also increase \\
\hline \multicolumn{3}{|l|}{ Supervised classification } \\
\hline Anderson et al. (2006) & RIPNet & Need for one model for each component; Also, different set of variables is used in each model \\
\hline Clarke et al. (2003) & Minimum Distance & Minimum distance classifier may be sensitive to variations on the data \\
\hline Clarke et al. (2006) & Maximum Likelihood & Hemorrhage and necrotic-core are similar according to the MR signals \\
\hline Hofman et al. (2006) & ANN, Bayes and K-NN & $\begin{array}{l}\text { The low number of pixels used for calcification may explain the worst results; Slight overlapping may also have } \\
\text { affected the results }\end{array}$ \\
\hline van Engelen et al. (2012) & Linear Discriminant & $\begin{array}{l}\text { Large number of input values; Difficults in differentiating fibrous tissue and necrotic core components; Validation on } \\
\text { in-vivo image is necessary }\end{array}$ \\
\hline van Engelen et al. (2012) & Linear Discriminant & Quite number of input values for each pixel is necessary for classification \\
\hline van 't Klooster et al. (2012) & Linear Discriminant & $\begin{array}{l}\text { Similarity of the signal intensities of calcifications close to the lumen with other components have contributed for the } \\
\text { poor results; Also, more images with calcifications should be included in the training step to overcome this problem }\end{array}$ \\
\hline
\end{tabular}

studies (Anderson et al., 2006; Clarke et al., 2006; Clarke et al., 2003; van Engelen et al., 2012; Hofman et al., 2006; van Engelen et al., 2012) to classify each pixel into a tissue type.

Although the aforementioned studies have proved to be effective on identifying atherosclerotic plaque components, some drawbacks must be highlighted. PDA provides constant values for the segmentation of atherosclerotic plaque components in ultrasound images (Lal et al., 2002). However, redefinition of the gray levels of the components could be necessary due to different gray levels among images acquired with different equipment. In addition, calcified plaques could harm the segmentation due to projected shadow that can precludes regions of interest (Molinari et al., 2010). The use of HU values in CTA images also presents drawbacks. Similarly to the problems of PDA, the values of HU may change with different datasets. In the study of de Weert et al. (2006) lipid-core comprised lipid, hemorrhage and thrombus components due to the difficulty of detecting thrombus and hemorrhage in MDCT images. Also, lipid-core with midly calcified area shown better results compared to those obtained with hard calcium. According to the authors, it was due to lower blooming effect of the calcium areas (de Weert et al., 2006).

Similarity of intensity values difficults the discrimination of plaque components. In the study of Vukadinovic et al. (2012) the overlapping of the HU values corresponding to the lipid and fibrous tissues difficults the distinction of these components. Similar difficults can be found in the study of Adame et al. (2004b) and van Engelen et al. (2012). Difficults in detecting lipid core close to lumen is also covered in another study of Adame et al. (2004a).

The main drawback of supervised classification is still the need for a training step to build a predictive model. In addition, complex models with a high number of input elements achieved similar results to those presented with simpler algorithms. Clustering has been proposed for image segmentation as a simple methodology based on less complex mathematical models and no need for a training step. In combination with image processing techniques, methodologies to partition similar regions of an image and refine the boundaries of these regions with active contour algorithms can improve the results of existing methods of characterization of atherosclerotic plaque components. Since clustering algorithms provide unsupervised classification, the identification of the categories belonging to the partitioned regions becomes a problem due to the lack of labels corresponding to each region.

The main limitations of the works and proposals surveyed in this article are summarized in Table 6.

Studies addressing the combination of spatial fuzzy C-Means algorithms with active contours for identifying the components on atherosclerotic plaques is expected to be considered in the future. Spatial fuzzy C-means proved to be effective to overcome the presence of noises in images and improve the cluster partitioning (Beevi, Sathik, Senthamaraikannan, \& Yasmin, 2010; Hassan, Chaudhry, Khan, \& Kim, 2012; Krinidis \& Chatzis, 2010; Li, Chui, Chang, \& Ong, 2011). Use of spatial fuzzy c-means algorithms could also overcome the problems with transitions of intensities values belonging to a same component. In addition, the use of Adaptive Resonance Theory (ART) models (Beale \& Jackson, 1990; SerranoGotarredona, Linares-Barranco, \& Andreou, 1998) such as Fuzzy-ART and Fuzzy-ARTMAP can also be considered as alternative methods to classify the regions corresponding to the atherosclerotic plaque components.

\section{Conclusion and future research}

Quantification of atherosclerotic plaque components has been addressed in studies assessing the risks associated with neurological events. The morphology of such plaques is a well established indicator to predict events such as transient ischemic attacks, amaurosis fugax and strokes. Several methodologies has been proposed to quantify atherosclerotic plaques in the most popular imaging diagnosis. Automatic identification of plaque components performed by 
computational algorithms provides an expedited diagnosis of possible neurological events. Thin fibrous cap, lipid rich-necrotic core and intraplaque hemorrhages were covered as the components associated with high risk for such event.

The automatic classification of the atherosclerotic plaques is a demanding challenge to the expert systems usage. An auxiliary diagnosis of the disease progression performed by these systems allows the medical doctors to identify the characteristics of the diseases as soon as possible and, consequently, to expedite the treatment planning. As previously mentioned, the features extracted from the atherosclerotic plaques represent a key point for developing machine learning based expert systems to recognize the atherosclerotic lesion type or determine whether it is symptomatic or asymptomatic.

Future studies addressing the improvement of the proposed computational algorithms could provide more accuracy in identifying the components and correctly delineating their boundaries. The overlapping of the main components associated with high risk for neurological events is still a problem in many studies dealing with the segmentation of atherosclerotic plaques. The main cause of this is the similar intensities of these components. Fuzzy C-Means algorithm proved to be effective in segmenting regions with similar intensities. In addition, spatial fuzzy C-Means allows to overcome the presence of local noise not treated with the traditional fuzzy C-Means. The combination of clustering with active contours could provide the correct identification and delineation of the components on atherosclerotic plaques.

Regarding the assessment of the atherosclerotic plaque from an expert systems development point of view, future researches addressing the classification of the disease progression are required to allow the development of medical decision-making systems for providing an efficient and complementary diagnosis for planning the adequate treatment. Since the accuracy of the automated classification depends on the information attained from the segmented structures in images, the extraction of the most relevant features from atherosclerotic plaques and their components is one of the most important task to be considered in future studies for allowing the accurate assessment of carotid diseases. Such features could allow the accuracy improvement of the most existing methodologies applied to the classification of the symptomatic or asymptomatic atherosclerosis. To the best of our knowledge, no one study presents an expert system for the assessment of the atherosclerotic lesion type based on the AHA classification standard. Therefore, as the main contribution that can be attained with the application of expert systems concerning the study of atherosclerotic plaques, we suggest the use of the most efficient classifiers addressed in the literature in order to perform the classification of the lesion type, particularly based on the referred standard.

\section{Acknowledgments}

This work was partially funded by Coordenação de Aperfeiçoamento de Pessoal de Nível Superior (CAPES), funding agency in Brazil under the PhD Grant with reference number 0543/13-6.

This work was funded by European Regional Development Funds (ERDF), through the Operational Programme 'Thematic Factors of Competitiveness'(COMPETE), and Portuguese Funds, through the Fundação para a Ciência e a Tecnologia (FCT), under the project: FCOMP-01-0124-FEDER-028160/PTDC/BBB- BMD/3088/2012.

\section{References}

Abdel-Dayem, A. R., \& El-Sakka, M. R. (2007). Fuzzy C-Means clustering for segmenting carotid artery ultrasound images. In M. Kamel, \& A. Campilho (Eds.), Image analysis and recognition (pp. 935-948). doi:10.1007/978-3-540-74260-9_83.

Acharya, U. R., Faust, O., Sree, S. V., Alvin, A. P. C., Krishnamurthi, G., Seabra, J. C. R. et al. (2011). Atheromatic: Symptomatic vs. asymptomatic classification of carotid ultrasound plaque using a combination of HOS, DWT \& texture. In Proceedings of the 2011 annual international conference of the ieee engineering in medicine and biology society (EMBS) (pp. 4489-4492). doi:10.1109/IEMBS.2011.6091113.
Acharya, U. R., Sree, S. V., Molinari, F., Saba, L., Nicolaides, A., Shafique, S., et al. (2012) Carotid ultrasound symptomatology using atherosclerotic plaque characterization: A class of Atheromatic systems. In Proceedings of the 2012 annual international conference of the ieee engineering in medicine and biology society (EMBS) (pp. 31993202). doi:10.1109/EMBC.2012.6346645.

Adame, I. M., van der Geest, R. J., Wasserman, B. A., Mohamed, M., Reiber, J. H. C. \& Lelieveldt, B. P. F. (2004a). Automatic plaque characterization and vessel wall segmentation in magnetic resonance images of atherosclerotic carotid arteries. In Medical imaging 2004 (pp. 265-273). International Society for Optics and Photonics, doi:10.1117/12.532471.

Adame, I. M., Van Der Geest, R. J., Wasserman, B. A., Mohamed, M. A., Reiber, J. H. C., \& Lelieveldt, B. P. F. (2004b). Automatic segmentation and plaque characterization in atherosclerotic carotid artery MR images. Magnetic Resonance Materials in Physics, Biology and Medicine, 16(5), 227-234. doi:10.1007/s10334-003-0030-8.

Anderson, R. W., Stomberg, C., Hahm, C. W., Mani, V., Samber, D. D., Itskovich, V. V., et al. (2006). Automated classification of atherosclerotic plaque from magnetic resonance images using predictive models. Bio Systems, 90(2), 456-466. doi:10.1016/ j.biosystems.2006.11.005.

Beale, R., \& Jackson, T. (1990). Neural computing: An introduction. Bristol, UK: IOP Publishing Ltd.

Beevi, S. Z., Sathik, M. M., Senthamaraikannan, K., \& Yasmin, J. H. J. (2010). A robust fuzzy clustering technique with spatial neighborhood information for effective medical image segmentation: An efficient variants of fuzzy clustering technique with spatial information for effective noisy medical image segmentation. In Proceedings of the 2010 second international conference on computing, communication and networking technologies (ICCNT) (pp. 1-8). IEEE. doi:10.1109/ICCCNT.2010 5591787.

Bezdek, J. C., Ehrlich, R., \& Full, W. (1984). FCM: The fuzzy c-means clustering algorithm. Computers E' Geosciences, 10(2), 191-203. doi:10.1016/0098-3004(84)90020-7.

Biasi, G. M., Mingazzini, P. M., Baronio, L., Piglionica, M. R., Ferrari, S. A., Elatrozy, T. S. et al. (1998). Carotid plaque characterization using digital image processing and its potential in future studies of carotid endarterectomy and angioplasty. Journal of Endovascular Surgery, 5(3), 240-246. doi:10.1583/1074-6218(1998)005<0240: CPCUDI $>2.0 . \mathrm{CO} ; 2$

Bluth, E. I., Kay, D., Merritt, C. R. B., Sullivan, M., Farr, G., Mills, N. L., et al. (1986) Sonographic characterization of carotid plaque: Detection of hemorrhage. American Journal of Roentgenology, 146(5), 1061-1065. doi:10.2214/ajr.146.5.1061.

Brenner, D. J., \& Hall, E. J. (2007). Computed tomography - an increasing source of radiation exposure. New England Journal of Medicine, 357(22), 2277-2284. doi:10.1056/ NEJMra072149.

Brigger, P., Hoeg, J., \& Unser, M. (2000). B-spline snakes: a flexible tool for parametric contour detection. IEEE Transactions on Image Processing, 9(9), 1484-1496. doi:10. $1109 / 83.862624$.

Bushberg, J. (2002). The essential physics of medical imaging (2nd ed.). Philadelphia, PA: Lippincott Williams \& Wilkins.

Cai, J.-M. (2002). Classification of human carotid atherosclerotic lesions with in vivo multicontrast magnetic resonance imaging. Circulation, 106(11), 1368-1373. doi:10.1161/01.CIR.0000028591.44554.F9.

Chan, T. F., \& Vese, L. A. (2001). Active contours without edges. IEEE Transactions on Image Processing, 10(2), 266-277. doi:10.1109/83.902291.

Christodoulou, C. I., Pattichis, C. S., Pantziaris, M., \& Nicolaides, A. (2003). Texture-based classification of atherosclerotic carotid plaques. IEEE Transactions on Medical Imaging, 22(7), 902-912. doi:10.1109/TMI.2003.815066.

Chu, B., Kampschulte, A., Ferguson, M. S., Kerwin, W. S., Yarnykh, V. L., O’Brien, K. D., et al. (2004). Hemorrhage in the atherosclerotic carotid plaque: a high-resolution MRI study. Stroke, 35(5), 1079-1084. doi:10.1161/01.STR.0000125856.25309.86.

Clarke, S. E., Beletsky, V., Hammond, R. R., Hegele, R. A., \& Rutt, B. K. (2006). Validation of automatically classified magnetic resonance images for carotid plaque compositional analysis. Stroke, 37(1), 93-97. doi:10.1161/01.STR.0000196985.38701.0c.

Clarke, S. E., Hammond, R. R., Mitchell, J. R., \& Rutt, B. K. (2003). Quantitative assessment of carotid plaque composition using multicontrast MRI and registered histology. Magnetic Resonance in Medicine, 50(6), 1199-1208. doi:10.1002/mrm.10618.

Dalager, M. G., Bøttcher, M., Andersen, G., Thygesen, J., Pedersen, E. M., Dejbjerg, L., et al. (2011). Impact of luminal density on plaque classification by CT coronary angiography. The International Journal of Cardiovascular Imaging, 27(4), 593-600. doi:10.1007/s10554-010-9695-Z.

Delsanto, S., Molinari, F., Giustetto, P., Liboni, W., Badalamenti, S., \& Suri, J. (2007). Characterization of a completely user-independent algorithm for carotid artery segmentation in 2D ultrasound images. IEEE Transactions on Instrumentation and Measurement, 56(4), 1265-1274. doi:10.1109/TIM.2007.900433.

Diethrich, E. B., Irshad, K., \& Reid, D. B. (2006). Virtual histology and color flow intravascular ultrasound in peripheral interventions. Seminars in Vascular Surgery, 19(3), 155-162. doi:10.1053/j.semvascsurg.2006.06.001.

El-Barghouty, N., Geroulakos, G., Nicolaides, A., Androulakis, A., \& Bahal, V. (1995) Computer-assisted carotid plaque characterisation. European Journal of Vascular and Endovascular Surgery, 9(4), 389-393. doi:10.1016/S1078-5884(05)80005-X.

Elatrozy, T., Nicolaides, A., Tegos, T., \& Griffin, M. (1998). The objective characterisation of ultrasonic carotid plaque features. European Journal of Vascular and Endovascular Surgery, 16(3), 223-230. doi:10.1016/S1078-5884(98)80224-4.

van Engelen, A., Niessen, W. J., Klein, S., Groen, H. C., Verhagen, H. J. M., Wentzel, J. J., et al. (2012). Multi-feature-based plaque characterization in ex vivo MRI trained by registration to 3D histology. Physics in Medicine and Biology, 57(1), 241-256. doi:10. 1088/0031-9155/57/1/241

Fukunaga, K., \& Hostetler, L. (1975). The estimation of the gradient of a density function, with applications in pattern recognition. IEEE Transactions on Information Theory, 21(1), 32-40. doi:10.1109/TIT.1975.1055330. 
Furie, K., Smimakis, S., Koroshetz, W., \& Kistler, J. (2004). Stroke due to large artery atherosclerosis. In K. L. Furie, \& P. J. Kelly (Eds.), Handbook of stroke prevention in clinical practice. In Current Clinical Neurology (pp. 151-165). Humana Press. doi:10. 1007/978-1-59259-769-7_10.

Geroulakos, G., Ramaswami, G., Nicolaides, A., James, K., Labropoulos, N., Belcaro, G. et al. (1993). Characterization of symptomatic and asymptomatic carotid plaques using high-resolution real-time ultrasonography. British Journal of Surgery, 80(10) 1274-1277. doi:10.1002/bjs.1800801016.

Goldman, L. W. (2007). Principles of CT and CT technology. Journal of Nuclear Medicine Technology, 35, 115-128. doi:10.2967/jnmt.107.042978.

de Graaf, M. A., Broersen, A., Kitslaar, P. H., Roos, C. J., Dijkstra, J., Lelieveldt, B. P. F., et al. (2013). Automatic quantification and characterization of coronary atherosclerosis with computed tomography coronary angiography: cross-correlation with inravascular ultrasound virtual histology. The International Journal of Cardiovascular Imaging, 29(5), 1177-1190. doi:10.1007/s10554-013-0194-X

Granada, J. F., Wallace-Bradley, D., Win, H. K., Alviar, C. L., Builes, A., Lev, E. I., et al. (2007). In vivo plaque characterization using intravascular ultrasound-virtual histology in a porcine model of complex coronary lesions. Arteriosclerosis, Thrombosis and Vascular Biology, 27(2), 387-393. doi:10.1161/01.ATV.0000253907.51681.0e.

Grogan, J. K., Shaalan, W. E., Cheng, H., Gewertz, B., Desai, T., Schwarze, G., et al. (2005) B-mode ultrasonographic characterization of carotid atherosclerotic plaques in symptomatic and asymptomatic patients. Journal of Vascular Surgery, 42(3), 435441. doi:10.1016/j.jvs.2005.05.033.

Hassan, M., Chaudhry, A., Khan, A., \& Kim, J. Y. (2012). Carotid artery image segmentation using modified spatial fuzzy c-means and ensemble clustering. Computer Methods and Programs in Biomedicine, 108(3), 1261-1276. doi:10.1016/j.cmpb.2012. 08.011

Herbert, C., Chandler, A., \& Dinsmore, R. (1995). A definition of advanced types of atherosclerotic lesions and a histological classification of atherosclerosis. Circulation, 92(5), 1355-1374. doi:10.1161/01.CIR.92.5.1355.

Hofman, J. M. A., Branderhorst, W. J., ten Eikelder, H. M. M., Cappendijk, V. C., Heeneman, S., Kooi, M. E., et al. (2006). Quantification of atherosclerotic plaque components using in vivo MRI and supervised classifiers. Magnetic Resonance in Medicine 55(4), 790-799. doi:10.1002/mrm.20828.

Hooda, H., Verma, O. P., \& Singhal, T. (2014). Brain tumor segmentation: A perfor mance analysis using k-means, fuzzy c-means and region growing algorithm. In Proceedings of the 2014 international conference on advanced communication control and computing technologies (icaccct) (pp. 1621-1626). doi:10.1109/ICACCCT.2014 7019383

Itskovich, V. V., Samber, D. D., Mani, V., Aguinaldo, J. G. S., Fallon, J. T., Tang, C. Y., et al. (2004). Quantification of human atherosclerotic plaques using spatially enhanced cluster analysis of multicontrast-weighted magnetic resonance images. Magnetic Resonance in Medicine, 52(3), 515-523. doi:10.1002/mrm.20154.

Kampschulte, A., Ferguson, M. S., Kerwin, W. S., Polissar, N. L., Chu, B., Saam, T., et al. (2004). Differentiation of intraplaque versus juxtaluminal hemorrhage/thrombus in advanced human carotid atherosclerotic lesions by in vivo magnetic resonance imaging. Circulation, 110(20), 3239-3244. doi:10.1161/01.CIR.0000147287.23741. $9 \mathrm{~A}$.

Karmonik, C., Basto, P., \& Morrisett, J. D. (2006). Quantification of carotid atherosclerotic plaque components using feature space analysis and magnetic resonance imaging. In Proceedings of the 28th annual international conference of the ieee engineering in medicine and biology society (embs'06): 1 (pp. 3102-3105). doi:10.1109 IEMBS.2006.260318.

Karmonik, C., Basto, P., Vickers, K., Martin, K., Reardon, M. J., Lawrie, G. M., et al. (2009). Quantitative segmentation of principal carotid atherosclerotic lesion components by feature space analysis based on multicontrast MRI at 1.5 T. IEEE Transactions on Biomedical Engineering, 56(2), 352-360. doi:10.1109/TBME.2008.2003100.

Kerwin, W., Xu, D., Liu, F., Saam, T., Underhill, H., Takaya, N., et al. (2007). Magnetic resonance imaging of carotid atherosclerosis: plaque analysis. Topics in Magnetic Resonance Imaging, 18(5), 371-378. doi:10.1097/rmr.0b013e3181598d9d.

Kerwin, W. S., Han, C., Chu, B., Xu, D., Luo, Y., Hwang, J.-N., et al. (2001). A quantitative vascular analysis system for evaluation of atherosclerotic lesions by MRI. In W. Niessen, \& M. Viergever (Eds.), Medical image computing and computer-assisted intervention (miccai) 2001. In Lecture Notes in Computer Science: 2208 (pp. 786-794). Springer Berlin Heidelberg. doi:10.1007/3-540-45468-3_94

Koenig, W., \& Khuseyinova, N. (2007). Biomarkers of atherosclerotic plaque instability and rupture. Arteriosclerosis, Thrombosis, and Vascular Biology, 27, 15-26. doi:10. 1161/01.ATV.0000251503.35795.4f.

Krinidis, S., \& Chatzis, V. (2010). A robust fuzzy local information C-Means clustering algorithm. IEEE Transactions on Image Processing, 19(5), 1328-1337. doi:10.1109/TIP. 2010.2040763.

Kyriacou, E., Pattichis, C. S., Pattichis, M. S., Mavrommatis, A., Panagiotou, S. Christodoulou, C. I., et al. (2006). Classification of atherosclerotic carotid plaques using gray level morphological analysis on ultrasound images. Artificial Intelligence Applications and Innovations, 204, 737-744. doi:10.1007/0-387-34224-9_87.

Kyriacou, E., Pattichis, M. S., Pattichis, C. S., Mavrommatis, A., Christodoulou, C. I. Kakkos, S., et al. (2009). Classification of atherosclerotic carotid plaques using morphological analysis on ultrasound images. Applied Intelligence, 30(1), 3-23. doi:10.1007/s10489-007-0072-0.

Lal, B. K. Hobson, R. W. Hameed, M., Pappas, P. J., Padberg, F. T., Jamil, Z., et al. (2006). Noninvasive identification of the unstable carotid plaque. Annals of Vascular Surgery, 20(2), 167-174. doi:10.1007/s10016-006-9000-8.

Lal, B. K., Hobson, R. W., Pappas, P. J., Kubicka, R., Hameed, M., Chakhtura, E. Y., et al (2002). Pixel distribution analysis of B-mode ultrasound scan images predicts histologic features of atherosclerotic carotid plaques. Journal of Vascular Surgery, 35(6), 1210-1217. doi:10.1067/mva.2002.122888.
Li, B. N., Chui, C. K., Chang, S., \& Ong, S. H. (2011). Integrating spatial fuzzy clustering with level set methods for automated medical image segmentation. Computers in Biology and Medicine, 41(1), 1-10. doi:10.1016/j.compbiomed.2010.10.007.

Li, X., \& Liu, D. (2007). Ultrasound image enhancement using dynamic filtering. In Proceedings of the 4th international conference on image and graphics (icig) (pp. 106109). doi:10.1109/ICIG.2007.43.

Liu, F., Xu, D., Ferguson, M. S., Chu, B., Saam, T., Takaya, N., et al. (2006). Automated in vivo segmentation of carotid plaque MRI with morphology-enhanced probability maps. Magnetic Resonance in Medicine, 55(3), 659-668. doi:10.1002/mrm.20814.

Loizou, C. P., Petroudi, S., Pantziaris, M., Nicolaides, A. N., \& Pattichis, C. S. (2014). An integrated system for the segmentation of atherosclerotic carotid plaque ultrasound video. IEEE Transactions on Ultrasonics, Ferroelectrics, and Frequency Control, 61(1), 86-101. doi:10.1109/TUFFC.2014.6689778.

Loizou, C. P., Petroudi, S., Pattichis, C. S., Pantziaris, M., Kasparis, T., \& Nicolaides, A. (2012). Segmentation of atherosclerotic carotid plaque in ultrasound video. In Proceedings of the 2012 annual international conference of the IEEE engineering in medicine and biology society (EMBS) (pp. 53-56). doi:10.1109/EMBC.2012. 6345869.

Lovett, J. K., Gallagher, P. J., Hands, L. J., Walton, J., \& Rothwell, P. M. (2004). Histological correlates of carotid plaque surface morphology on lumen contrast imaging. Circulation, 110(15), 2190-2197. doi:10.1161/01.CIR.0000144307.82502.32.

Ma, Z., Tavares, J. M. R., Jorge, R. N., \& Mascarenhas, T. (2010). A review of algorithms for medical image segmentation and their applications to the female pelvic cavity. Computer Methods in Biomechanics and Biomedical Engineering, 13(2), 235-246. doi:10.1080/10255840903131878.

Mendis, S., Puska, P., \& Norrving, B. (2011). Global atlas on cardiovascular disease prevention and control. World Health Organization.

Michailovich, O., \& Tannenbaum, A. (2007). Segmentation of medical ultrasound images using active contours. In Proceedings of the 2007 IEEE international conference on image processing (ICIP): 5. doi:10.1109/ICIP.2007.4379878. (pp. V - 513$\mathrm{V}-516)$

Moftah, H., Elmasry, W., El-Bendary, N., Hassanien, A., \& Nakamatsu, K. (2012). Evaluating the effects of k-means clustering approach on medical images. In Proceedings of the 12th international conference on intelligent systems design and applications (ISDA) (pp. 455-459). doi:10.1109/ISDA.2012.6416581.

Molinari, F., Liboni, W., Giustetto, P., Pavanelli, E., Marsico, A., \& Suri, J. S. (2010). Carotid plaque characterization with contrast-enhanced ultrasound imaging and its histological validation. Journal for Vascular Ultrasound, 34(4), 175-184.

Molinari, F., Liboni, W., Pavanelli, E., Giustetto, P., Badalamenti, S., \& Suri, J. S. (2007). Accurate and automatic carotid plaque characterization in contrast enhanced 2-D ultrasound images. In Proceedings of the 29th annual international conference of the ieee engineering in medicine and biology society (EMBS) (pp. 335-338). doi:10.1109/ IEMBS.2007.4352292

Nair, A. (2002). Coronary plaque classification with intravascular ultrasound radiofrequency data analysis. Circulation, 106(17), 2200-2206. doi:10.1161/01.CIR. 0000035654.18341.5E

NASCET (1991). Beneficial effect of carotid endarterectomy in symptomatic patients with high-grade carotid stenosis. New England Journal of Medicine, 325(7), 445453. doi:10.1056/NEJM199108153250701.

Pedro, L. M., Pedro, M. M., Gonçalves, I., Carneiro, T. F., Balsinha, C., Fernandes e Fernandes, R., et al. (2000). Computer-assisted carotid plaque analysis: characteristics of plaques associated with cerebrovascular symptoms and cerebral infarction. European Journal of Vascular and Endovascular Surgery, 19(2), 118-123. doi:10.1053/ejvs.1999.0952.

Saam, T., Cai, J., Ma, L., Cai, Y.-Q., Ferguson, M. S., Polissar, N. L., et al. (2006). Comparison of symptomatic and asymptomatic atherosclerotic carotid plaque features with in vivo MR imaging. Radiology, 240(2), 464-472. doi:10.1148/radiol.2402050390.

Saam, T., Ferguson, M. S., Yarnykh, V. L., Takaya, N., Xu, D., Polissar, N. L., et al. (2005). Quantitative evaluation of carotid plaque composition by in vivo MRI. Arteriosclerosis, Thrombosis, and Vascular Biology, 25(1), 234-239. doi:10.1161/01.ATV. 0000149867.61851.31.

Salem, M. K., Bown, M. J., Sayers, R. D., West, K., Moore, D., Nicolaides, A., et al. (2014). Identification of patients with a histologically unstable carotid plaque using ultrasonic plaque image analysis. European Journal of Vascular and Endovascular Surgery, 48(2), 118-125. doi:10.1016/j.ejvs.2014.05.015.

Salem, M. K., Sayers, R. D., Bown, M. J., West, K., Moore, D., Nicolaides, A., et al. (2012). Patients with recurrent ischaemic events from carotid artery disease have a large lipid core and low GSM. European Journal of Vascular and Endovascular Surgery, 43(2), 147-153. doi:10.1016/j.ejvs.2011.11.008.

Santos, A. M. F, dos Santos, R. M. Castro, P. M. A. Azevedo, E. Sousa, L, \& Tavares, J. M. R. (2013). A novel automatic algorithm for the segmentation of the lumen of the carotid artery in ultrasound B-mode images. Expert Systems with Applications, 40(16), 6570-6579. doi:10.1016/j.eswa.2013.06.003.

Schadé, J. P. (2006). The Complete Encyclopedia of Medicine \& Health. Foreign Media Books

Schulte-Altedorneburg, G., Droste, D. W., Haas, N., Kemény, V., Nabavi, D. G., Füzesi, L., et al. (2000). Preoperative B-mode ultrasound plaque appearance compared with carotid endarterectomy specimen histology. Acta Neurologica Scandinavica, 101(3), 188-194. doi:10.1034/j.1600-0404.2000.101003188.x.

Seeger, J. M., Barratt, E., Lawson, G. A., \& Klingman, N. (1995). The relationship between carotid plaque composition, plaque morphology, and neurologic symptoms. The Journal of Surgical Research, 58(3), 330-336. doi:10.1006/jsre.1995.1051.

Serfaty, J. M., Chaabane, L., Tabib, A., Chevallier, J. M., Briguet, A., \& Douek, P. C. (2001) Atherosclerotic plaques: classification and characterization with T2-weighted high-spatial-resolution MR imaging- an in vitro study. Radiology, 219(2), 403-410. doi:10.1148/radiology.219.2.r01ma15403. 
Serrano-Gotarredona, T., Linares-Barranco, B., \& Andreou, A. G. (1998). Adaptive resonance theory microchips: circuit design techniques. Norwell, MA, USA: Kluwer Academic Publishers.

Sodickson, A., Baeyens, P. F., Andriole, K. P., Prevedello, L. M., Nawfel, R. D., Hanson, R., et al. (2009). Recurrent CT, cumulative radiation exposure, and associated radiation-induced cancer risks from CT of adults. Radiology, 251(1), 175-184. doi:10.1148/radiol.2511081296.

Steffen, C. M., Gray-Weale, A. C., Byrne, K. E., \& Lusby, R. J. (1989). Carotid artery atheroma: ultrasound appearance in symptomatic and asymptomatic vessels. The Australian and New Zealand Journal of Surgery, 59(7), 529-534. doi:10.1111/j. 1445-2197.1989.tb01625.x.

Sun, B., Giddens, D. P., Long, R., Taylor, W. R., Weiss, D., Joseph, G., et al. (2006). Characterization of coronary atherosclerotic plaque using multicontrast MRI acquired under simulated in vivo conditions. Journal of Magnetic Resonance Imaging, 24, 833841. doi:10.1002/jmri.20687.

Sun, B., Giddens, D. P., Long, R., Taylor, W. R., Weiss, D., Joseph, G., et al. (2008). Automatic plaque characterization employing quantitative and multicontrast MRI. Magnetic Resonance in Medicine, 59, 174-180. doi:10.1002/mrm.21279.

Sztajzel, R. (2005). Ultrasonographic assessment of the morphological characteristics of the carotid plaque. Swiss Medical Weekly, 135(43/44), 635-643. doi:2005/ 43/smw-11038.

Takaya, N., Yuan, C., Chu, B., Saam, T., Underbill, H., Cai, J., et al. (2006). Association between carotid plaque characteristics and subsequent ischemic cerebrovascular events: A prospective assessment with MRI - Initial results. Stroke, 37(3), 818-823. doi:10.1161/01.STR.0000204638.91099.91

Tang, H., van Walsum, T., Hameeteman, R., Schaap, M., van der Lugt, A., van Vliet, L. J., et al. (2012). Lumen segmentation of atherosclerotic carotid arteries in CTA. In Proceedings of the 9th IEEE international symposium on biomedical imaging (ISBI) (pp. 274-277). doi:10.1109/ISBI.2012.6235537.

Toussaint, J. F., LaMuraglia, G. M., Southern, J. F., Fuster, V., \& Kantor, H. L. (1996). Magnetic resonance images lipid, fibrous, calcified, hemorrhagic, and thrombotic components of human atherosclerosis in vivo. Circulation, 94(5), 932-938. doi:10.1161/ 01.CIR.94.5.932.

van Engelen, A., Niessen, W. J., Klein, S., Groen, H. C., Verhagen, H. J. M., Wentzel, J. J., et al. (2012). Supervised in-vivo plaque characterization incorporating class label uncertainty. In Proceedings of the 9th IEEE international symposium on biomedical imaging (pp. 246-249). doi:10.1109/ISBI.2012.6235530.

van 't Klooster, R., Naggara, O., Marsico, R., Reiber, J. H. C., Meder, J. F., Van Der Geest, R. J. et al. (2012). Automated versus manual in vivo segmentation of carotid plaque MRI. American Journal of Neuroradiology, 33, 1621-1627. doi:10.3174/ajnr.A3028.

Vukadinovic, D., Rozie, S., Van Gils, M., Van Walsum, T., Manniesing, R., Van Der Lugt, A., et al. (2012). Automated versus manual segmentation of atherosclerotic carotid plaque volume and components in CTA: Associations with cardiovascular risk factors. International Journal of Cardiovascular Imaging, 28(4), 877-887. doi:10.1007/s10554-011-9890-6.
Vukadinovic, D., van Walsum, T., Manniesing, R., Rozie, S., van der Lugt, A., \& Niessen, W. J. (2011). Region based level set segmentation of the outer wall of the carotid bifurcation in CTA. In SPIE medical imaging. International Society for Optics and Photonics (pp. 79623P-79623P-8). doi:10.1117/12.878114.

Vukadinovic, D., Walsum, T. V., Rozie, S., Weert, T. D., Manniesing, R., Lugt, A. V. D., et al. (2009). Carotid artery segmentation and plaque quantification in CTA. In Proceedings of the 2009 IEEE international symposium on biomedical imaging: From nano to macro (ISBI '09) (pp. 835-838). doi:10.1109/ISBI.2009.5193182.

Warlow, C. (1991). Mrc european carotid surgery trial: interim results for symptomatic patients with severe (70-99\%) or with mild (0-29\%) carotid stenosis. The Lancet, 337(8752), 1235-1243. doi:10.1016/0140-6736(91)92916-P.

Watanabe, Y., Nagayama, M., Sakata, A., Okumura, A., Amoh, Y., Ishimori, T., et al. (2014). Evaluation of fibrous cap rupture of atherosclerotic carotid plaque with thin-slice source images of Time-of-Flight MR Angiography. Annals of Vascular Diseases, 7(2), 127-133. doi:10.3400/avd.oa.13-00101.

de Weert, T. T., Ouhlous, M., Meijering, E., Zondervan, P. E., Hendriks, J. M., van Sambeek, M. R., et al. (2006). In vivo characterization and quantification of atherosclerotic carotid plaque components with multidetector computed tomography and histopathological correlation. Arteriosclerosis, thrombosis, and vascular biology, 26(10), 2366-2372. doi:10.1161/01.ATV.0000240518.90124.57.

Widder, B., Paulat, K., Hackspacher, J., Hamann, H., Hutschenreiter, S., Kreutzer, C., et al (1990). Morphological characterization of carotid artery stenoses by ultrasound duplex scanning. Ultrasound in Medicine \& Biology, 16(4), 349-354. doi:10.1016 0301-5629(90)90064-J.

Wiebers, D. O., Feigin, V. L., \& Brown, R. (2006). Handbook of Stroke. Board Review Series (2nd). Philadelphia, PA: Lippincott Williams \& Wilkins.

Wintermark, M., Jawadi, S. S., Rapp, J. H., Tihan, T., Tong, E., Glidden, D. V., et al. (2008). High-resolution CT imaging of carotid artery atherosclerotic plaques. American Journal of Neuroradiology, 29(5), 875-882. doi:10.3174/ajnr.A0950.

Xu, D. X. D., Hwang, J.-N. H. J.-N., \& Yuan, C. Y. C. (2001). Atherosclerotic plaque segmentation at human carotid artery based on multiple contrast weighting MR images. In Proceedings of the 2001 international conference on image processing: 2 (pp. 849-852). doi:10.1109/ICIP.2001.958627.

Yin, H., \& Liu, D. (2009). Lateral resolution enhancement of ultrasound image using neural networks. In Proceedings of the 3rd international conference on bioinformatics and biomedical engineering (ICBBE) (pp. 1-4). doi:10.1109/ICBBE.2009.51630110.

Yuan, C. (2002). Identification of fibrous cap rupture with magnetic resonance imaging is highly associated with recent transient ischemic attack or stroke. Circulation, 105(2), 181-185. doi:10.1161/hc0202.102121.

Yuan, C., Mitsumori, L. M., Ferguson, M. S., Polissar, N. L., Echelard, D., Ortiz, G., et al. (2001). In vivo accuracy of multispectral magnetic resonance imaging for identifying lipid-rich necrotic cores and intraplaque hemorrhage in advanced human carotid plaques. Circulation, 104(17), 2051-2056. doi:10.1161/hc4201.097839.

Zhao, L., \& Jolesz, F. (2014). Navigation with the integration of device tracking and medical imaging. In F. A. Jolesz (Ed.), Intraoperative imaging and image-guided therapy (pp. 259-276). Springer New York. doi:10.1007/978-1-4614-7657-3_18. 